\title{
Transposons: Moving Forward from Preclinical Studies to Clinical Trials
}

\author{
Jaitip Tipanee, Thierry VandenDriessche, ${ }^{1,2, *}$ and Marinee K. Chuah ${ }^{1,2, *}$ \\ ${ }^{1}$ Department of Gene Therapy and Regenerative Medicine, Free University of Brussels (VUB), Brussels, Belgium; ${ }^{2}$ Center for Molecular and Vascular Biology, \\ Department of Cardiovascular Sciences, University of Leuven, Leuven, Belgium.
}

Transposons have emerged as promising vectors for gene therapy that can potentially overcome some of the limitations of commonly used viral vectors. Transposons stably integrate into the target cell genome, enabling persistent expression of therapeutic genes. Transposons have evolved from being used as basic tools in biomedical research to bona fide therapeutics. Currently, the most promising transposons for gene therapy applications are derived from Sleeping Beauty $(S B)$ or piggyBac $(P B)$. Stable transposition requires co-delivery of the transposon DNA with the corresponding transposase gene, mRNA, or protein. Stable transposition efficiency can be substantially increased by using "next-generation" transposon systems that combine codon-usage optimization with hyper-activating mutations in the SB or PB transposases. By virtue of their relatively large capacity, gene therapy applications with relatively large therapeutic transgenes, such as full-length dystrophin, can now be envisaged. The authors and others have shown that efficient and stable gene transfer can be achieved with these next-generation transposons in several clinically relevant primary cells, such as CD34 ${ }^{+}$hematopoietic stem/progenitor cells, $\mathrm{T}$ cells, and mesenchymal and myogenic stem/progenitor cells that are amenable for ex vivo transfection. Alternatively, in vivo transposon gene delivery has been explored using non-viral vectors or nanoparticles or in combination with viral vectors. The therapeutic potential of these $S B$ - and $P B$-based transposons has been demonstrated in preclinical models that mimic the cognate human diseases. However, there are still challenges impeding clinical translation of transposons pertaining mainly to the typical limiting efficiencies of most non-viral transfection methods and the intrinsic DNA toxicity. Nevertheless, it is particularly encouraging that transposons have now been used in gene therapy clinical trials. In particular, transposon-engineered $\mathrm{T}$ cells expressing chimeric antigen receptors are starting to yield promising results in patients with hematological malignancies.

Keywords: transposon, piggyBac, Sleeping Beauty, Tol2, chimeric antigen receptor, stem cells, iPS

\section{INTRODUCTION}

GENE THERAPY, DEFINED AS the introduction of genes into the patient's target cells for therapeutic purposes, has shown remarkable progress in recent years. ${ }^{1-5}$ Compelling proof of therapeutic efficacy has been demonstrated primarily by gene addition, whereby copies of the genes are introduced de novo into the cells without interfering with the endogenous gene copies. Successful gene therapy has been achieved by ex vivo gene delivery into the desired target cells. To achieve this, the target cells are first isolated from the patients (or healthy donors) and genetically engineered ex vivo. Subsequently, these gene-modified cells are transplanted into the patients in an autologous or allogeneic setting. Several gene therapy products, based on gene-modified CD34 ${ }^{+}$hematopoietic stem/progenitor cells (HSC) or $\mathrm{T}$ cells, have obtained marketing authorization approval by the regulatory authorities. ${ }^{5-7}$ Alternatively, in vivo gene delivery based on the direct transfer of genes administered through the vasculature or locally in the target tissue has proven successful for a number of disease targets, including hemophilia, retinal diseases, or metabolic disorders.

${ }^{*}$ Correspondence: Drs. Thierry VandenDriessche and Marinee K. Chuah, Free University of Brussels (VUB), Department of Gene Therapy and Regenerative Medicine, Faculty of Medicine and Pharmacy, Building D, room D365, Laarbeeklaan 103, B-1090 Brussels, Belgium. E-mail: thierry.vandendriessche@vub.ac.be; marinee.chuah@vub.ac.be

(C) Jaitip Tipanee et al. 2017; Published by Mary Ann Liebert, Inc. This is an Open Access article distributed under the terms of the Creative Commons Attribution License, which permits unrestricted use, distribution, and reproduction in any medium, provided the original work is properly cited. 
This prompted regulatory approval of a gene therapy product (Glybera ${ }^{\circledR}$ ) for lipoprotein lipase deficiency. ${ }^{8}$ These advances relied on the use of viral vectors, mostly $\gamma$-retroviral, lentiviral, or adeno-associated virus (AAV) vectors. These vectors are replication defective. However, they retain the favorable properties of their parental viruses by enabling efficient cell entry and delivery of the therapeutic gene cargo into the desired target cells. Despite these advances, viral vectors can provoke inflammatory or adaptive immune responses after in vivo gene delivery that curtail long-term gene expression and raise safety concerns. ${ }^{9}$ In addition, integrating viral vectors (i.e., $\gamma$-retroviral) were found to trigger leukemia due to the activation of cellular proto-oncogenes. ${ }^{10-12}$ In the case of lentiviral vectors, clonal dominance has been reported in clinical trials. ${ }^{13}$ Finally, the large-scale manufacturing of these viral vectors in cell lines poses a formidable and rather costly challenge that hampers clinical translation and delays pivotal Phase III trials.

To overcome some of the limitations of these viral vector systems, non-viral vectors have been used instead. ${ }^{14}$ Typically, these non-viral vectors systems are fully synthetic and do not rely on any viral component or mammalian cell-dependent manufacturing. This dramatically reduced the manufacturing costs while alleviating at least some of the immune concerns associated with the use of viral vectors. Though transfection of naked DNA into cells is notoriously inefficient, a plethora of non-viral transfection methods have been developed to improve gene transfer efficacy. Their purpose is to prevent DNA from degradation and to facilitate the uptake of the therapeutic DNA in the target cells. Typically, the therapeutic genes are transferred in forms of plasmids specifically tailored to maximize gene expression in the intended somatic target cells. However, even in optimal conditions of high transfection efficiencies, expression of the gene of interest by non-viral vectors is only short-term due to its degradation and gradual dilution of the episomal DNA in dividing target cells. This justifies the development of an efficient, integrating non-viral vector system based on mobile DNA element or transposons that stably integrate the therapeutic gene in the target cell chromosomes. ${ }^{15-19}$ Consequently, non-viral transfection of transposons combines some of the advantages of viral vectors (i.e., sustained expression of the therapeutic gene) with those of non-viral vectors (i.e., reduced immunogenicity and cost-effective manufacturing). Nevertheless, the incoming and transfected DNA by itself can activate the innate immune system..$^{20,21}$
Transposable elements are divided into two categories: retrotransposons and DNA transposons. Transposable elements can alter the genome of the host cells through insertions, duplications, deletions, and translocations. ${ }^{22,23}$ Retrotransposons are described as mobile elements that employ an RNA intermediate that is first reverse transcribed into a complementary single-stranded (c) DNA strand by a reverse transcriptase encoded by the retrotransposon. Subsequently, the single-stranded DNA is converted into a double-stranded DNA that then integrates into the host genome. This socalled "replicative mechanism" yields several new copies of retrotransposons expanding throughout the target genome over evolutionary time. Retrotransposons are categorized into many subtypes according to the DNA sequences of the long terminal repeats and its open reading frames. ${ }^{24}$ Retrotransposons were employed to enable transgene integration into the target cell DNA, in some cases relying on adenoviral delivery. ${ }^{25}$ Nevertheless, retrotransposons can actively spread through the human genome and create genomic instability in different ways, even potentially contributing to disease. ${ }^{26}$ Alternatively, DNA transposons translocate via a "non-replicative mechanism," whereby two Terminal Inverted Repeats (TIRs) are recognized and cleaved by a transposase enzyme, releasing the cognate DNA transposons with free DNA ends. The excised DNA transposons then integrate into a new genomic region where target sites are recognized and cut by the same transposase. This cut-and-paste mechanism usually duplicates DNA target sites upon insertion, leaving target site duplications (TSDs). ${ }^{19}$ The most recent transposition of DNA transposons present in the human genome was estimated to have occurred 80 million years ago during evolution. ${ }^{27}$ Hence, endogenous DNA transposons are therefore considered to be silent in mammalian genomes, obviating concerns associated with transposon spreading and genome instability as in the case of retrotransposons.

\section{DNA TRANSPOSONS}

The typical architecture of DNA transposons includes a gene encoding a transposase for transposition, flanked by two TIRs. DNA transposons can be classified into diverse families, which differ in terms of target site recognition, TSDs, TIRs, and transposon DNA sequences. Some of these DNA transposon subgroups are employed for human gene therapy to achieve sustained gene expression of the therapeutic transgene. ${ }^{15,19}$ 


\section{Sleeping Beauty (SB)}

The Sleeping Beauty (SB) transposon belongs to the Tc1/mariner superfamily, which constitutes a large group of transposable elements that are widespread in several eukaryotic organisms. Salmonid transposons exhibit high DNA sequence homology to the Caenorhabditis elegans Tc1 transposon and were first identified in fish genomes in $1994 .^{28}$ However, these transposons were initially silent, primarily because of the accumulation of inactivating mutations in transposase genes. Thus, the functional transposase gene was originally reconstructed by "reverse evolution" based on consensus DNA sequence of different transposons derived from eight fish species by Ivics et al. ${ }^{29}$ They showed, for the first time that this resurrected transposase exerted transposition activity specifically to TIRs of salmonid transposons. ${ }^{29}$

Originally, the $S B$ transposon was about $1.6 \mathrm{~kb}$ in total size and was mainly composed of two inverted repeat/direct repeats (IR/DRs) flanking the gene encoding a 360-amino acid SB transposase (SBase). Two transposase-binding regions, referred as direct repeats (DRs), are present in each IR/DR, and both of them are important for transposase recognition. ${ }^{19}$ In contrast to the right IR/DR, the left IR/DR contains a $11 \mathrm{bp}$ DNA sequence similar to 3'-half of the DR, namely the HDR motif, which significantly enhances transposition rate. ${ }^{30}$

The SBase contain several distinctive structural motifs, including two DNA-binding helix-turnhelix (HTH) domains, consisting of PAI and RED subdomains, and catalytic domains harboring AspAsp-Glu (DDE) residues for DNA hydrolysis. ${ }^{31} \mathrm{~A}$ GRPR-like sequence is located between two HTH domains and is highly conserved among Tc1/ mariner membered transposases. ${ }^{32}$ This GRPR motif is responsible for Hin invertase-DNA interaction. ${ }^{33}$ The cut-and-paste transposition mechanism begins with multiple interactions between the transposase subunits and specific DNA motifs, that is, binding of PAI together with the RED subdomains to the DRs, and binding of the individual PAI subdomain to HDR. This ensures the specificity of the SB transposase activity toward the cognate $S B$ transposon. This is followed by formation of tetrameric structure between the SB transposase and the $S B$ transposon, allowing DNA and enzymes to be in close proximity for further DNA cleavage. ${ }^{30}$ The synaptic transposome complex is also stabilized by the high mobility group box 1 protein (HMGB1). ${ }^{34}$ The two strands of the transposon are cleaved by hydrolysis generating 5 'trinucleotide overhangs at both ends of transposon. The SBase also cleaves the genomic DNA at a TA dinucleotide consensus site to create the integration site for transposition. The cleaved transposon is then ligated to the integration site ends, resulting in the generation of a TA dinucleotide duplication. ${ }^{31}$ Doublestranded breaks with non-identical 3 '-trinucleotide overhangs at the original integration site can be repaired through multiple pathways, such as DNA-PKdependent end-joining, Ku-independent end-joining, and homology-dependent gap repair, which may result in DNA deletion or transposon footprints depending on the actual DNA repair mechanism. ${ }^{35}$

To generate a tool for gene delivery, the original SB system was converted into a two-component system by separating the two IR/DRs flanking the gene of interest from the SBase gene onto two different plasmids (Fig. 1). Typically, these two plasmids are co-delivered into target cells, and transposition occurs through a cut-and-paste mechanism, just as with the native transposon. ${ }^{19} \mathrm{Ex}-$ pressing the SBase from an expression plasmid carries the risk that this plasmid may potentially integrate. Moreover, even non-integrated expression plasmids can give rise to low-level sustained SBase expression. Sustained SBase expression may result in the continuous transposon mobilization and integration. Consequently, this may result in steadily increasing integrated transposon copies per cell that may in turn augment the risk of insertional oncogenesis. As a safer alternative, it is also possible to deliver the SBase as an mRNA. ${ }^{36-38}$ The SBase mRNA is typically transfected into the cells by electroporation. An improved approach of retrovirus particle-mediated mRNA transfer (designated as RMT) was recently developed that allowed for transient and dose-controlled expression of SBase. ${ }^{39,40}$ Interestingly, RMT provides a mean to support efficient transposition while preventing overt cell damage. In this case, the short-term presence of the SBase would create a window of opportunity, enabling transposition while substantially reducing the risk of insertional oncogenesis.

Several improvements have been reported to enhance SB transposition efficiency, including the generation of hyperactive transposase mutants. First-generation hyperactive SBase, called SB11, was generated by introduction of various amino acid mutations into the original transposase (SB10), leading to a relatively modest approximately threefold higher transposition efficiency. ${ }^{41}$ Similarly, consensus amino acids of transposases from several species were incorporated into the SBase, yielding more hyperactive versions such as HSB5 $^{42}$ and SB100X. ${ }^{43}$ What distinguishes SB100X in particular from the other SB transposases 


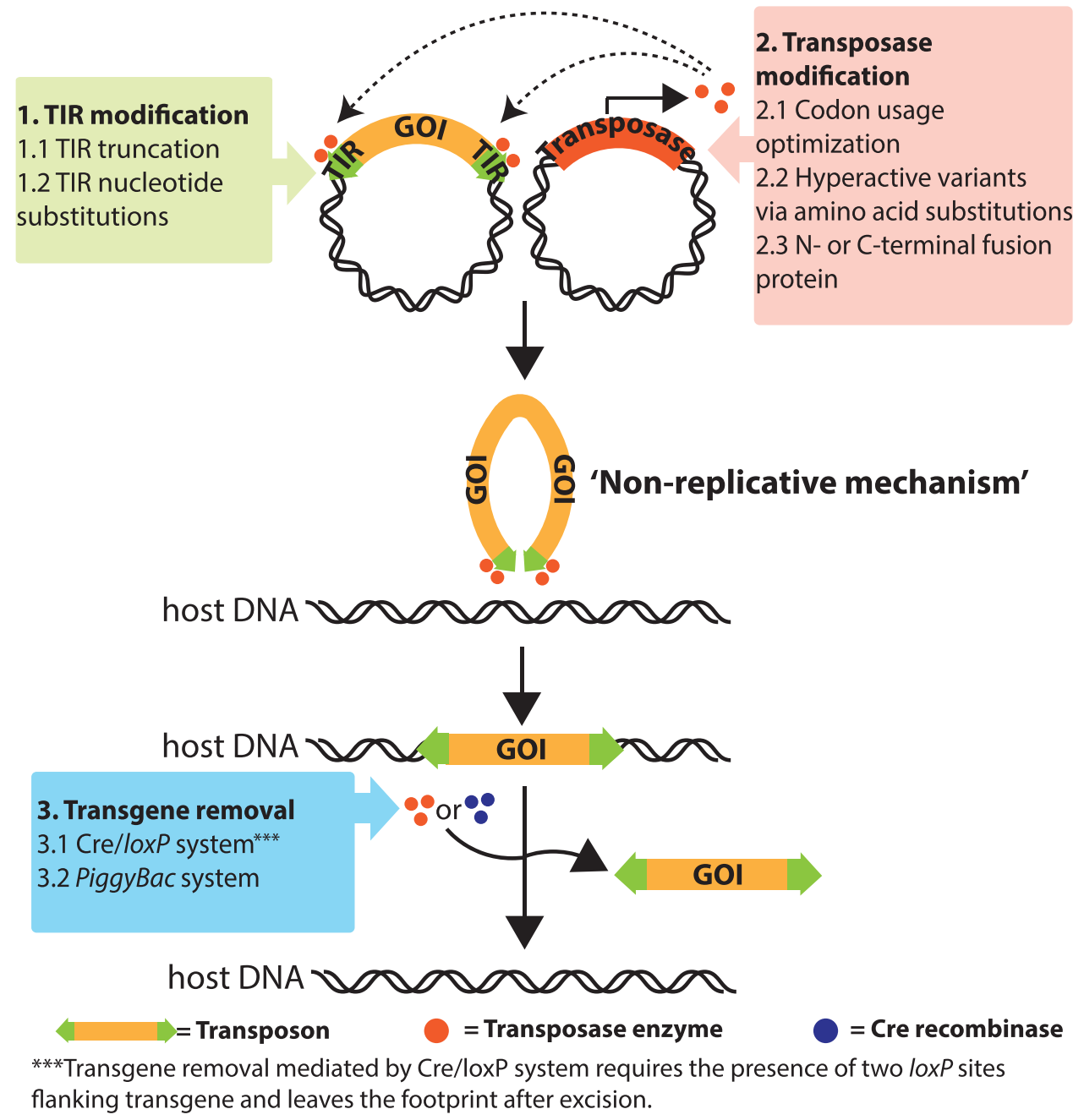

Figure 1. Schematic representation of two-component transposon system for gene delivery and improvements. Terminal inverted repeats (TIRs) and transposase gene of Sleeping Beauty (SB), piggyBac (PB), and Tol2 transposons are located in two different plasmids. The gene of interest (GOI) is flanked by two TIRs. Upon co-delivery of transposon-GOI and transposase vectors into host cells, transposases bind to TIRs for cleavage and form the transpososome complex with the transposon containing the GOI. The transposase subsequently introduces double-strand breaks and inserts the transposon with the GOI into the target site in the host DNA through a non-replicative (cut-and-paste) transposition mechanism, resulting in transposon integration. Several strategies have been developed to improve transposition activity, including TIR and transposase modification. The use of transposon/transposases also allows for specific excision of GOls by supplying the transposase transiently. In the case of $P B$, the excision is seamless, leaving no genetic trace.

is that it was generated by in vitro molecular evolution and selection. SB100X was 100 -fold more efficient in transposon mobilization using a marker rescue assay than the original SB10. The authors were the first to demonstrate the superiority of this SB100X system in clinically relevant primary cells, such as $\mathrm{CD}^{+} 4^{+} \mathrm{HSC}$, muscle stem/progenitor cells, mesenchymal and mesoangioblasts (MABs), hepatocytes, and induced pluripotent stem cell (iPS)-derived myogenic cells. SB100X ${ }^{43-45}$ significantly outperformed HSB5 ${ }^{46,47}$ and is considered the most hyperactive SBase to date. The authors have recently combined several other hyper-activating mutations within SB100X, but they did not further increase the overall trans- position efficacy (VandenDriessche and Chuah, unpublished observations).

Generally, there is no strict limitation with respect to the transgene size, at least up to $8 \mathrm{~kb}$. In contrast to transposons, viral vectors have a more limited packaging capacity. Nevertheless, transposition efficiency does decrease with increased transgene size beyond $8 \mathrm{~kb} .{ }^{41}$ However, it is possible to expand the transgene capacity of transposons by using a so-called sandwich configuration whereby the expression cassette is flanked with two complete pairs of IR/DRs in inverted orientation. ${ }^{48}$ Consequently, this transposon payload can be further increased to at least $10 \mathrm{~kb}$ DNA while maintaining a relatively efficient transposition. 
Additionally, the payload capacity of $S B$ transposon can be increased up to $12 \mathrm{~kb}$ when $S B$ transposon is delivered in the context of a herpes simplex virus (HSV) amplicon vector ${ }^{49}$ or a helper-dependent adenoviral vector. ${ }^{47}$

\section{PiggyBac (PB)}

The piggyBac $(P B)$ transposon was first identified and isolated from the cabbage looper moth Trichoplusia ni genome. ${ }^{50,51}$ The original $P B$ transposon is 2,475 bp in length and encodes a 594-amino acid PB transposase (PBase) gene. The $P B$ transposon is flanked by a 311 bp $5^{\prime}$ end and 235 bp $3^{\prime}$ end, each containing TIR sequences. ${ }^{52}$ Similar to SBase, PBase contains a catalytic unit harboring a characteristic DDE/D motif, ${ }^{53}$ which interacts with $\mathrm{Mg}^{2+}$ ions during transposition. ${ }^{54}$ The C-terminal region of the PBase possesses a $\mathrm{Zn}^{2+}$-binding "plant homeodomain" (PHD) finger with conserved cysteine residues. ${ }^{55}$ The $\mathrm{PB}$ system also undergoes a typical cut-and-paste transposition reaction, as in the case of SB, whereby the PBase recognizes the two TIRs of the $P B$ transposon. Unlike $S B$ transposons, the $P B$ transposon preferential genomic integration site contains a TTAA consensus motif. ${ }^{19}$ The binding of the PBase to the transposon is followed by the 3 '-strand excision of DNA, leaving the free 3 '-OH group, which further forms the hairpin structure with 5'-TTAA overhangs of the transposon. The hairpin transposome complex is then released from the genome and binds to the site of integration in which the TTAA consensus sequence is present. The hairpin chemical bonds are subsequently cleaved by the transposase enzyme, and free $3^{\prime}-\mathrm{OH}$ attack the target site to generate TTAA overhangs in the target genome. The TTAA overhangs of the $P B$ transposon and the target genome eventually anneal to complete the transposition reaction. Through this mechanism, the $P B$ transposon system also leads to TGDs upon completion of the transposition process. However, in contrast to $S B, P B$ transposon does not leave $3 \mathrm{bp}$ footprints at the original transposition site, since the two $5^{\prime}$ overhangs generated by PBase excision are perfectly complementary. ${ }^{52}$

A two-component PB platform has been developed and demonstrated, analogous to SB, yielding relatively robust stable gene transfer efficiencies in several independent studies ${ }^{19}$ (Fig. 1). Additionally, the PBase has been modified to boost transposition activity in mammalian systems. The first strategy consisted of engineering the PBase DNA sequence by codon optimization for use in mammalian cells. For instance, mouse (m) and human (h) codon-optimized PBases were derived from the original PBase ${ }^{56,57}$ and are expressed at higher levels in mammalian cells, significantly improving the overall transposition efficacy. Alternatively, novel amino acid mutations that were screened for increased transposition efficacy were introduced into the PBase. These mutants were generated by using error-prone polymerase chain reaction (PCR), which were subsequently validated in yeast cells. Ultimately, this resulted in a hyperactive PBase (hyPBase) that contained seven amino acid substitutions that synergistically enhanced transposition efficiency. ${ }^{58}$ Comparative in vitro studies indicated that the hyPBase outperformed the mPBase by 10 -fold, at least in mouse ES cells. ${ }^{58}$ Similarly, a 20-fold increase in liver-directed expression has been shown with the hyPBase compared to when the mPBase was employed. ${ }^{59}$ In an attempt to increase transposition further, the TIRs of the $P B$ transposons were engineered. The $5^{\prime}$ and $3^{\prime}$ TIRs were trimmed to retain only those functional regions that were sufficient for transposition, thus reducing the total size of the $P B$ vectors. ${ }^{60}$ The smallest TIRs were defined as having only 40 and $57 \mathrm{bp}$ of $3^{\prime}$ and $5^{\prime}$ IRs (referred as " $I R_{\text {micro" }}$ ", respectively, and they exhibited relatively robust transposition activity compared to the native IRs. ${ }^{61}$ Alternatively, IR variants bearing nucleotide substitutions were generated by random PCR amplification to investigate the effects of substituted nucleotides on PB transposition. The $5^{\prime}$ IR harboring T53C and C146T mutations (referred as " $I R_{\text {mut }}$ ") substantially improved transposition when used in combination with the hPBase. ${ }^{56}$ Though side-by-side comparison in vivo demonstrated an increase of transgene integration mediated by $\mathrm{IR}_{\text {micro }}$ relative to original IRs, this was not the case with $\mathrm{IR}_{\text {mut }}{ }^{59,62} \mathrm{In}$ terms of foreign DNA payload, in vitro studies of human and murine models showed that the $\mathrm{PB}$ system is capable of carrying up to $100 \mathrm{~kb}$ transgenes. ${ }^{59,63,64}$ The PB platform was adapted to accommodate even $200 \mathrm{~kb}$ of bacterial artificial chromosomes for animal transgenesis. ${ }^{64}$ Delivery of such large transgenes is not possible with viral vectors. Nevertheless, transfection efficiencies decrease with increased transgene size, warranting selective enrichment of those cells that had undergone stable transposition. ${ }^{44}$ Taken together, the attributes of $P B$ transposon make them particularly attractive for non-viral vector-based gene transfer. ${ }^{19,65-67}$

\section{Tol2}

The Tol2 transposable element belongs to the $h A T$ (hobo/Ac/Tam3) superfamily and was discovered in 
1996 in the medaka fish (Orizyas latipes) genome as the first autonomous transposon in vertebrate hosts. ${ }^{68}$ Tol2 is approximately $4.7 \mathrm{~kb}$ in size and is composed of a gene encoding the transposase flanked by $12 \mathrm{bp}$ TIRs. Tol 2 mRNA contains four exons, which give rise to several transposase isoforms of which the 649-amino acid isoform is the most active variant. ${ }^{69}$ Tol2 transposition generates a 8 bp TGD at the integration site. ${ }^{70}$ Tol2 transposon is able to deliver a genetic cargo up to $10-11 \mathrm{~kb}$ in several mammalian cells without compromising transposition efficiency. ${ }^{71-74}$ Moreover, the Tol2two-component system has been used successfully to generate transgenic animals such as mouse, chicken, frog ${ }^{75-79}$ and particularly zebrafish models. ${ }^{72,77,80,81}$ Like SB and PB, the Tol2 system enables sustained transgene expression after gene delivery, thereby providing the advantage of being suitable vector for gene therapy ${ }^{72,73}$ (Fig. 1). To improve the efficacy of Tol2 system for gene transfer, Tol2 transposase was codon-optimized for enhancement of transposase expression in mammalian systems, including mouse and human. ${ }^{69,82}$ Furthermore, the original Tol2 transposon was truncated to carry a $200 \mathrm{bp} 5^{\prime}$ end and a $150 \mathrm{bp} 3^{\prime}$ end to minimize the overall size of the Tol2 mobile element while retaining the transposition capacity (referred as "minimal Tol2"). This minimal Tol2 resulted in an approximately threefold increased transposition activity compared to the full-length Tol2 system. ${ }^{61}$

Taken together, $S B, P B$, and Tol2 transposons are considered relatively efficient non-viral vectors for stable integration of the gene of interest into the target cell genomes, enabling long-term gene expression, even of relatively large transgenes. However, different transposon platforms do not function at comparable efficiencies, especially in the context of primary human cells or ultimately in clinical trials. The relative transposition efficiency of these different transposon systems varies depending on several confounding variables, including the cell type, transfection efficiency, DNA concentration, transposon/transposase ratio, and read-out (i.e., mobilization or rescue, gene expression, and antibiotic resistance). Consequently, head-to-head comparisons under the same conditions with rigorous controls, including inactive transposases, are necessary to determine the relative efficacy of different transposon systems. For instance, head-tohead comparative analysis showed that the SB100X and $\mathrm{mPBase}$ transposase-based systems were more robust than Tol2, at least in HeLa cells. ${ }^{83}$ In addition, transposition in human CD34 ${ }^{+}$HSC showed that SB100X outperformed mPBase. ${ }^{83}$

\section{Ex vivo transposon delivery}

Several approaches have been used to deliver transposons into the desired target cells to achieve sustained transgene expression. The introduction of transposons into host cells has to overcome the challenges of the intrinsic physiological and cellular barriers, including cell membranes and the instability of naked DNA due to DNA degradation. The delivery methods that have been employed to transfect transposons in vitro can be classified into DNA-chemical complexes (i.e., calcium phosphate, liposome, polymers, and nanoparticles), electroporation, and microinjection. Calcium phosphate transfection allows the formation of DNA- $\mathrm{CaCl}_{2}$ complexes that bind to the cell surface and are engulfed by host cells through endocytosis. This method is relatively simple compared to other techniques, and is therefore used in easy-to-transfect cell lines, yielding high transposition efficiency. ${ }^{84,85}$ Similarly, chemical-based transfection can also be achieved by cationic liposomes and polyethyleneimine (PEI), ${ }^{86-89}$ which effectively binds to the transposon/transposase DNA and facilitates their interaction with the target cell membranes. However, most clinically relevant primary target cells such as CD34 ${ }^{+}$HSC, immune cells, and muscle cell progenitors are relatively refractory to these types of transfection methods. Electroporation has been used as an alternative transfection method for transposons in primary cells. It results in reversible membrane pore formation that facilitates transposon/ transposase DNA uptake into the primary target cells, including CD34 ${ }^{+} \mathrm{HSC},{ }^{43} \mathrm{~T}$ cells, ${ }^{73}$ mesenchymal stem/progenitor cells, ${ }^{45}$ iPS,${ }^{45}$ myoblasts, ${ }^{45,66}$ MABs, ${ }^{44}$ and iPS-derived progenitors (VandenDriessche, unpublished observations). Nevertheless, one limitation of electroporation is that it results in significant cell mortality, particularly when DNA is used. Magnetofection, which relies on magnetic nanoparticles delivered into cells using magnetic fields, is sometimes used for gene transfer into difficult-to-transfect cells and offers similar transfection efficiency to electroporation, depending on the target cells.

\section{HSC}

because of their self-renewal potential and ability to differentiate into different lympho-hematopoietic lineages, HSC are promising target cells for gene therapy. Initially, an early generation $S B$ transposon (i.e., SB10) had been used to deliver genes stably into HSC, though the overall efficiencies were relatively modest, and no evidence was provided to demonstrate efficient hematopoietic reconstitution and multi-lineage marking in vivo. ${ }^{90}$ 
Later, it was demonstrated, for the first time, that transposon-modified $\mathrm{CD} 34^{+} \mathrm{HSC}$ contributed to efficient and sustained multi-lineage gene marking in vivo using the SB100X system. ${ }^{43}$ This demonstrated the potential of the hyperactive SB100X system for stable gene transfer in bona fide HSC that outperformed the early generation SB systems, creating new perspectives for HSC-based gene therapy. This was later independently confirmed in other studies. ${ }^{91,92}$ It was then shown that the ability to use transposons to delivery genes stably into $\mathrm{CD}^{+} 4^{+} \mathrm{HSC}$ can be extended to the PB system, though SB100X was more efficient. ${ }^{83}$ However, comparative studies with the latest generation hyPB would be required to determine which of the two systems is ultimately the most efficient. As a clinically relevant application, transposons were used to drive $\beta$-globin expression in patient-derived hematopoietic progenitors, resulting in a reduced sickling phenotype in red blood cells in vitro. ${ }^{93}$

\section{Mesenchymal and myogenic stem/progenitor cells}

Myoblasts are self-renewing adult muscle progenitor cells that can undergo terminal myogenic differentiation into skeletal muscle fibers ${ }^{94,95}$ that have been explored for cell therapy of muscle disorders. SB100X-mediated gene transfer was optimized to improve transposition efficiency up to 10 -fold in myoblasts compared to SB11. ${ }^{45}$ Correspondingly, the SB platform was used to deliver therapeutic genes stably, including human dysferlin $^{96}$ and micro-dystrophin, ${ }^{97}$ a truncated dystrophin variant, in myoblasts that stably expressed the corresponding transgenes and retained their myogenic characteristics upon engraftment in mice. ${ }^{96,97}$ Similarly, the PB system was also validated in myoblasts ${ }^{44,98}$ at least in vitro.

SB- or PB-based transposon-based gene transfer enabled stable gene integration in mesenchymal stem cells (MSCs). ${ }^{45,99-103}$ In particular, successful transgene transposition in MSCs was demonstrated using SB100X technology, which resulted in up to 10-fold higher transposition efficiency compared to the SB11 system. The genetically engineered MSCs maintained the ability to undergo osteogenic, myogenic, and adipogenic differentiation after electroporation with the SB100X or PB transposon system. ${ }^{45,99}$ The engineered MSC efficiently expressed coagulation factor IX (FIX), indicating that it is a suitable platform for production of secreted proteins. ${ }^{45}$ Recently, adipose-derived MSCs secreting interferon gamma were generated using the hyPBase system as a basis for cancer immunotherapy in murine models. ${ }^{103}$
MABs are capable of penetrating blood vessels during intra-arterial delivery, in contrast to myoblasts, which can only be delivered directly in the muscle tissue itself. MABs can undergo myogenic differentiation to promote muscle regeneration in dystrophic animal models. ${ }^{104-106}$ They can readily be engineered using transposon, resulting in persistent transgene expression in vivo upon transplantation in mouse models. ${ }^{89}$ By virtue of their capacity to accommodate large inserts, it has been shown that $P B$ transposons were able to deliver and express the full-length codon-optimized human dystrophin cDNA $(\sim 11.1 \mathrm{~kb})$ in dystrophic MABs when used in combination with the hyPBase. ${ }^{44}$ The main advantage is that all of the functional domains were retained in contrast to when truncated dystrophins were employed, but the overall efficiency was reduced due to the intrinsic DNA toxicity after electroporation.

\section{iPS cells}

iPS cells can be generated by de novo expression of the quintessential reprogramming factors Oct4, Sox2, Klf4, and c-Myc. ${ }^{107}$ Both $S B$ - and $P B$-based transposons have been employed to express these reprogramming factors and successfully reprogram mouse ${ }^{108-110}$ bovine,${ }^{111,112}$ porcine,${ }^{113}$ and human ${ }^{110,114,115}$ fibroblasts into iPS cells. Direct comparative analysis between hyPBase- and SB100X-mediated gene transfer showed that both systems displayed comparable reprogramming efficiency. ${ }^{109}$ One particular advantage of using transposons for iPS generation is that the reprogramming cassette can be excised using Cre/loxP, clustered regularly interspaced short palindromic repeats/CRISPR-associated protein-9 nuclease (CRISPR/Cas9) systems, or reintroduction of the transposase ${ }^{108,116}$ (Fig. 2). Consequently, so-called transgene-free or genetically unmodified iPS cells can be generated that cannot re-express any potentially oncogenic reprogramming genes. In addition, this also minimizing potential concerns associated with insertional oncogenesis. The PB system generally restores the site of integration without duplicating or adding footprint in host genome upon removal of the reprogramming cassette in contrast to when $\mathrm{SB}$ or Cre/loxP technology was employed. This constitutes a "seamless" excision strategy for the generation of genetically unmodified iPS cells. ${ }^{115}$

Transposons have also been used to introduce functional gene copies in patient-derived iPS cells containing defective genes. For instance, SBmediated ectopic expression of micro-utrophin in dystrophic iPS-derived skeletal muscle progenitors 
a

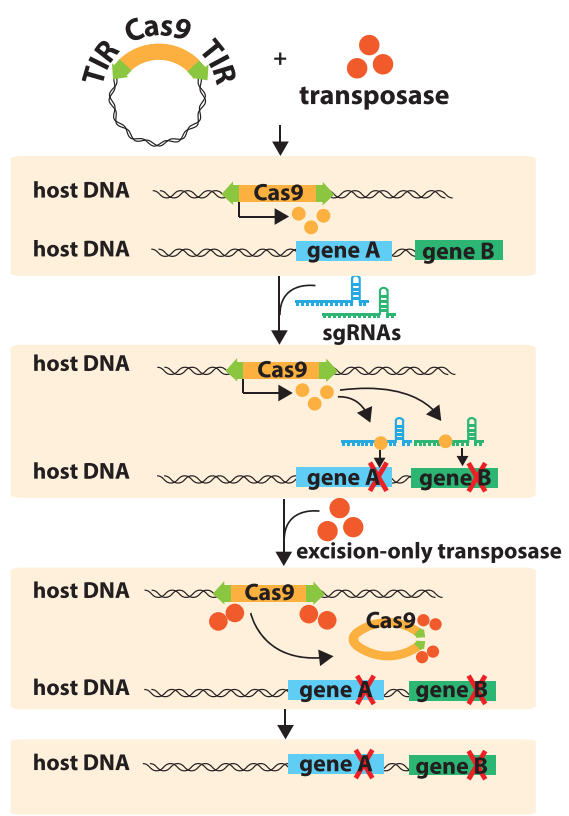

b

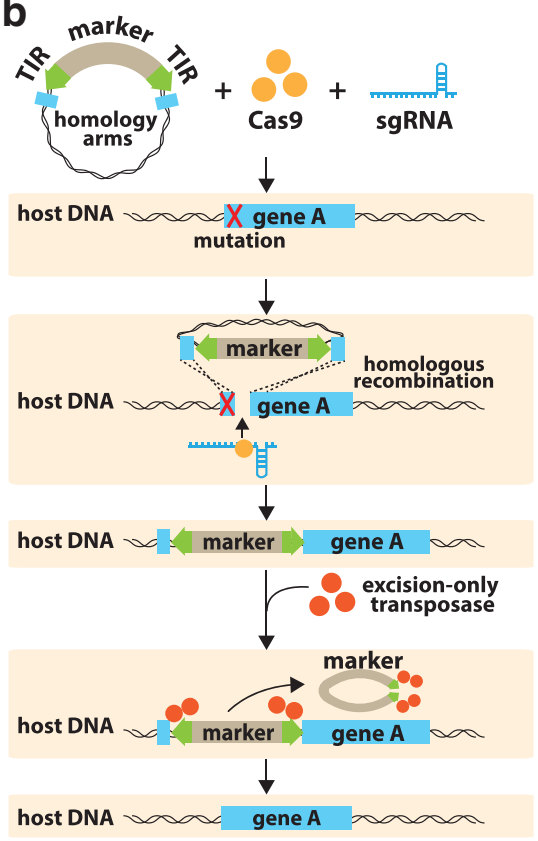

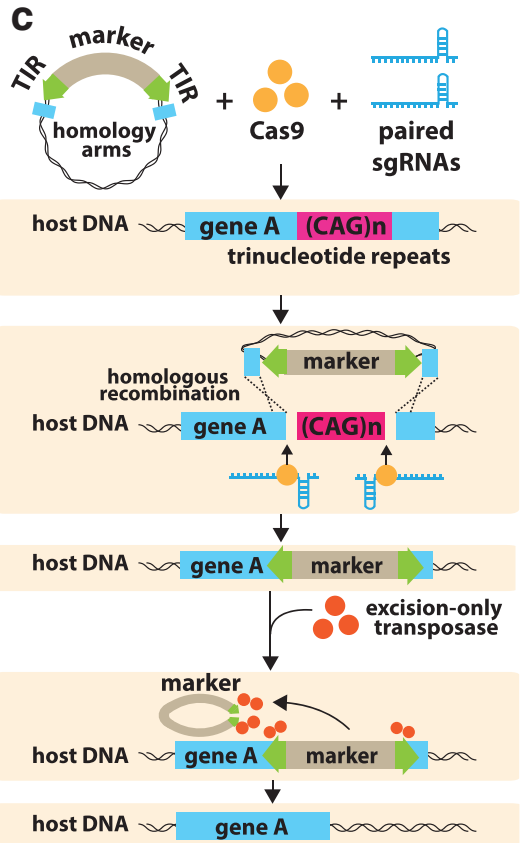

Figure 2. Schematic representation of possible strategies of combined PB-CRISPR/Cas9 for genome editing with seamless excision. (a) Gene disruption strategy. A Cas9 expression cassette in $P B$ transposon vector ( $P B$-Cas9) is co-delivered with PB transposase (PBase) for stable expression of Cas9 nuclease. Subsequently, single or multiplex single-guide RNAs (sgRNAs) are transfected into host cells expressing Cas 9 for targeted gene disruption. (b) Gene correction strategy. DNA sequence containing defective mutations are exposed to homologous DNA arms for gene correction. $P B$ transposons harboring a selectable marker ( $P B$-Marker) flanked with wild-type homologous DNA sequences of target genes are co-delivered with plasmids expressing Cas9 and sgRNA targeting homologous DNA integration; Cas9 activity leads to sgRNA-mediated DNA cleavage. This further allows homologous recombination between PB-Marker and host DNA at the defective mutated region, contributing to target gene correction. (c) Large genomic deletion. DNA sequence containing disease-associated trinucleotide repeats are exposed to homologous DNA arms for large genomic deletion. PB-Marker flanked with wild-type homologous DNA sequences of target genes are co-delivered with plasmids expressing Cas9 and paired sgRNAs targeting both ends of the expanded trinucleotide repeats. Cas9 activity gives rise to excision of trinucleotide repeats, and the presence of the $P B$-Marker in the host genome ensures successful homologous recombination between the $P B$-Marker and host DNA following genomic deletion. Eventually, $P B$ expression cassette in all three strategies can be removed by transient expression of PBase without leaving any genetic footprints.

restored the underlying muscle pathology by contributing to dystrophin-glycoprotein complex formation. ${ }^{117}$ This can be achieved following transplantation of transposon-modified iPS-derived muscle progenitors that resulted in improved muscle contraction strength. Additionally, the authors and others have shown that transposons can be used to coax the differentiation of iPS cells into their relevant differentiated cells. For instance, transposon-based ectopic expression of myogenic transcription factors such as PAX3 and MYOD1 induced the differentiation of iPS cells into muscle cell progenitors. ${ }^{45,118}$

Gene editing of an aberrant allele in iPS cells can be achieved following co-delivery of a $P B$ transposon carrying a correct donor sequence together with a site-specific designer nuclease, such as a transcription activator-like effector nuclease (TALEN), zinc finger nuclease (ZFN), or CRISPR/Cas9 (Fig. 2). The sequence-specific double-strand DNA break facilitates homology-directed integration of the donor DNA at this target locus. Subsequent tran- sient excision-only PBase activity allows for the seamless excision of selectable marker genes that are part of the targeting cassette. ${ }^{119}$ This hybrid platform was previously used for gene editing in iPS cells derived from patients with sickle cell disease caused by a $\beta$-globin $(H B B)$ gene mutation or Huntington's disease caused by trinucleotide repeat expansions in the huntingtin gene. ${ }^{120-122}$

\section{Primary T-lymphocytes}

Immunotherapy based on redirected $\mathrm{T}$ cells expressing chimeric antigen receptors (CARs) holds great promise for cancer therapy. ${ }^{123}$ The first use of transposons for $\mathrm{T}$ cell engineering was based on the use of SB10 to target the CD19 antigen. Transposon-modified redirected $\mathrm{T}$ cells efficiently mediated specific target cell lysis and cytokine production upon the exposure to CD19-positive leukemia cells and contributed to potent tumor remission in a murine model xenografted with tumor cells expressing CD19. ${ }^{124,125}$ Subsequently, SB100X for CAR T cell generation exerted superior 
transposition activity (fourfold higher) compared to SB11 and retained efficient target cell lysis. ${ }^{126}$ SB-based CAR T cells were also successfully generated to treat acute lymphocytic leukemia ${ }^{127}$ and sarcomas. ${ }^{128-130}$ Similarly, $P B$ transposons were explored to generate CAR T cells redirected against different antigens and cancer types, ${ }^{131-133}$ including myelomonocytic leukemia,${ }^{134}$ breast cancer, ${ }^{135}$ and cholangiocarcinoma. ${ }^{136}$ Tol2-mediated CD19specific CAR expression in T-lymphocytes was recently demonstrated to suppress B cell lymphoma progression both in vitro and in vivo. ${ }^{73}$ Comparative analysis indicated that $\mathrm{PB}$ was more robust than SB11 and Tol2 systems. ${ }^{137}$

\section{In vivo transposon delivery}

Transposon-mediated gene transfer has also been validated for in vivo transfection. Typically, for liver-directed gene delivery, transposon and transposase constructs were administered by hydrodynamic (HD) injection that involves rapid delivery of large volumes of DNA solutions. ${ }^{138} \mathrm{HD}$ transfection of transposons resulted in sustained gene expression in liver and kidney. ${ }^{59,62,139-142}$ In the absence of an active transposase, expression declined, perhaps due, at least in part, to epigenetic silencing, indicating that genomic integration was required to circumvent this and achieve stable expression. Moreover, in vivo stable inducible gene expression system can be established by transposon technology, allowing temporal control of transgene expression. ${ }^{143}$

HD-based transposon gene transfer has been utilized to direct long-term therapeutic gene expression in various inherited and acquired genetic disease models, including hemophilia $\mathrm{A}^{144,145}$ and $\mathrm{B},{ }^{141,146}$ sickle cell disease ${ }^{147}$ mucopolysaccharidosis (MPS) ${ }^{148-150}$ type I tyrosinemia, ${ }^{151}$ type I diabetes, ${ }^{152}$ familial hypercholesterolemia (FH) ${ }^{153}$ and type I Crigler-Najjar syndrome ${ }^{154}$ (Table 1). $S B$ transposon in conjunction with wild-type SBase or first-generation hyperactive SBase such as SB11 and HSB5/17 were initially used and were sufficient to enhance therapeutic gene expression and correct deficient phenotypes. For example, insulin expression mediated by native SB system significantly restored blood glucose to normal levels following glucose infusion in diabetic mice. ${ }^{152}$ In particular, hemophilia $B$ treatment in mouse and canine models can be achieved by standard $\mathrm{SB}^{141}$ or HSB $5^{146}$ platforms to alleviate bleeding diathesis and sustain physiological FIX levels. ${ }^{146}$ SB technology for in vivo gene therapy in MPS and hemophilia A was also validated and led to phenotypic correction following gene delivery. ${ }^{145,148,}, 149,155$
However, transgene expression dramatically decreased over time because of the subsequent development of immune response against therapeutic gene products. ${ }^{148}$ To overcome this limitation, immunomodulatory agents such as gadolinium chloride, and cyclophosphamide were used. ${ }^{148,156}$ Alternatively, the immunosuppressive enzyme indoleamine 2,3dioxygenase (IDO) ${ }^{144}$ was employed to boost immune tolerance and prolong transgene expression in mouse models. In a recent study in MPS mice, transposition efficacy of transgene gene mediated by SB100X was 15-fold more efficient than with the SB11 system and contributed to persistent therapeutic gene expression levels for 1 year. ${ }^{156}$ In addition, the SB100X technology encoding low-density and very-low-density lipoprotein receptors contributed to long-term therapeutic effect in FH mice. ${ }^{153}$ Therefore, the SB100X system holds great promise for further development of in vivo gene therapy to enhance transposition efficacy and stable expression of therapeutic genes.

Comparative studies indicated that hyPBase contributed to a threefold increase of transposition efficiency compared to the SB100X system, at least in one experimental model system. ${ }^{157} P B$ transposons were successfully used to correct von Willebrand's disease (vWD), ${ }^{158}$ unilateral ureteral obstruction (UUO), ${ }^{159,160}$ and hemophilia ${ }^{161}$ (Table 2). It has been demonstrated that $P B$ transposons efficiently directed expression of insulin-like growth factor-1 receptor (IGF-1R) and glutathione transferase isozyme A4 (GSTA4) and subsequently attenuate renal fibrosis formation, one of the major deficient symptoms in mice with UUO. ${ }^{159,160} \mathrm{In}$ addition to wild-type PBase, ${ }^{162}$ hyPBase system mediated long-term expression of blood clotting factor VIII (FVIII) and FIX and correct coagulation defects in hemophilia A and B mice, respectively. ${ }^{59,62,163}$ Comparative analysis in the hemophilia B murine model indicated that the hyPBase was more efficient than mPBase, resulting in higher stable FIX expression levels. ${ }^{59,62}$ PB-mediated expression of FIX and FVIII was long lasting (>300 days) and did not induce an immunological reaction. ${ }^{59,62,162}$

As a clinically feasible approach for transposonbased gene therapy, HD injection can be assisted by catheterization for local delivery of DNA in larger animal models such as pig, ${ }^{164} \mathrm{dog},{ }^{165,166}$ and monkey. ${ }^{60}$ It remains to be seen whether this method could eventually be applied safely and efficaciously in a clinical setting, given that hepatotoxicity ensued following HD injection. In some cases, immune suppression (e.g., with gadolinium chloride $\left[\mathrm{GdCl}_{3}\right]$ ) was warranted to prevent immune responses against the transgene product, as 
Table 1. Sleeping Beauty (SB) transposon application in gene therapy research

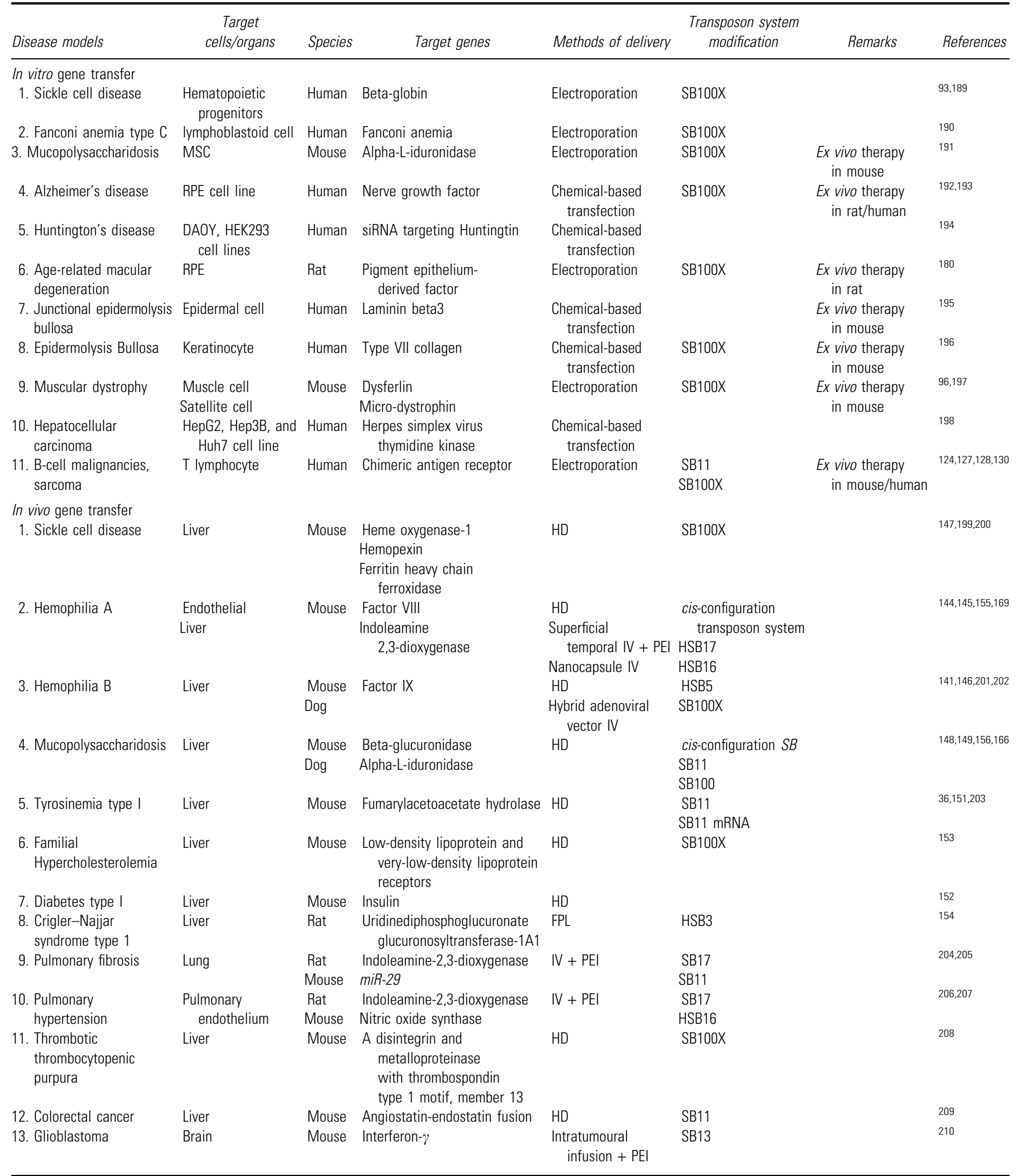

If not specifically indicated, two-component $S B$ transposon system was used in conjunction with SB10.

FPL, fusogenic galactose-terminated F-glycoprotein of the Sendai virus; HD, hydrodynamic injection; HSC, hematopoietic stem cell; IV, intravenous injection; MSC, mesenchymal stem cell; RPE, retinal pigment epithelium; PEI, polyethylenimine. 
Table 2. PiggyBac (PB) transposon application in gene therapy research

\begin{tabular}{|c|c|c|c|c|c|c|c|}
\hline Disease models & $\begin{array}{c}\text { Target } \\
\text { cells/organs }\end{array}$ & Species & Target genes & $\begin{array}{c}\text { Methods of } \\
\text { delivery }\end{array}$ & $\begin{array}{l}\text { Transposon system } \\
\text { modification }\end{array}$ & Remarks & References \\
\hline \multicolumn{8}{|l|}{ In vitro gene transfer } \\
\hline 1. Sickle cell disease & iPS cell & Human & Beta-globin & Electroporation & $\begin{array}{l}\text { hyPBase } \\
\text { Use in combination } \\
\text { with TALEN }\end{array}$ & & 120 \\
\hline 2. $\beta$-thalassemia & iPS cell & Human & Beta-globin & Electroporation & $\begin{array}{l}\text { hyPBase } \\
\text { Use in combination } \\
\quad \text { with CRISPR/Cas9 }\end{array}$ & & 121 \\
\hline 3. $\alpha 1$-antitrypsin deficiency & iPS cell & Human & Alpha-1 antitrypsin & Electroporation & $\begin{array}{l}\text { hyPBase } \\
\text { Use in combination } \\
\text { with ZFN }\end{array}$ & & 211 \\
\hline 4. Muscular dystrophy & MAB & Dog & Micro-dystrophin & Electroporation & hyPBase & & 44 \\
\hline $\begin{array}{l}\text { 5. Myeloid and lymphoid } \\
\text { malignancies, HER2-positive } \\
\text { solid tumor }\end{array}$ & T Lymphocyte & Human & $\begin{array}{l}\text { Chimeric antigen } \\
\text { receptor }\end{array}$ & Electroporation & hyPBase & & $132-135,212$ \\
\hline \multicolumn{8}{|l|}{ In vivo gene transfer } \\
\hline 1. Hemophilia A & Liver & Mouse & Factor VIII & $\mathrm{HD}$ & hyPBase & & 162,163 \\
\hline 2. Hemophilia B & Liver & Mouse & Factor IX & $\mathrm{HD}$ & $\begin{array}{l}\text { mPBase } \\
\text { hyPBase } \\
\mathbb{I}_{\text {mut }} \\
\mathbb{I}_{\text {micro }}\end{array}$ & & 59,213 \\
\hline 3. von Willebrand disease & Liver & Mouse & von Willebrand factor & $\mathrm{HD}$ & hyPBase & & 158 \\
\hline 4. Unilateral ureteral obstruction & Kidney & Mouse & $\begin{array}{l}\text { Insulin-like growth } \\
\text { factor-1 receptor } \\
\text { Glutathione transferase } \\
\text { isozyme A4 }\end{array}$ & HD & & & 159,160 \\
\hline 5. Cervical and ovarian solid tumor & Tumor & Mouse & $\begin{array}{l}\text { Herpes simplex virus } \\
\text { thymidine kinase }\end{array}$ & $\begin{array}{l}\text { Intratumoral } \\
\text { injection }+ \text { PEI }\end{array}$ & & & 214,215 \\
\hline
\end{tabular}

If not specifically indicated, two-component $P B$ transposon system was used in conjunction with wild-type PBase.

CRISPR/Cas9, clustered regularly interspaced short palindromic repeats/CRISPR-associated protein-9 nuclease; iPS, induced pluripotent stem cell; MAB, mesoangioblast; TALEN, transcription activator-like effector nucleases; ZFN, zinc finger nuclease.

in the case of $\alpha$-L-iduronidase (IDUA) and $\beta$ glucuronidase $\left(G U S B^{166}\right)$. Long-term FIX expression was also seen in the canine model (in the presence of $\mathrm{GdCl}_{3}$ ). Sustained therapeutic IDUA, GUSB, and FIX expression levels were able to be attained after SB transposition in dogs, which is an important step toward future clinical translation. As an alternative, PEI transfection has been explored as an alternative to HD in order to mediate in vivo transposon delivery through intravenous infusion into mice but was at least 100 -fold less efficient compared to HD delivery. ${ }^{167}$ Recently, in vivo administration of nanoparticles carrying transposons has emerged as an efficient method to deliver transgenes into circulating $\mathrm{T}$ cells in mice, and in situ engineered $\mathrm{T}$ cells exhibited similar activity to conventional engineered $\mathrm{T}$ cells generated by ex vivo gene transfer. ${ }^{168}$ Nanoparticles represents a potentially attractive technology to deliver transposons into liver sinusoidal endothelial cells. ${ }^{169}$ Intramuscular electroporation of transposons in murine models resulted in transient and localized transgene expression. ${ }^{89}$ To overcome the intrinsic challenges of non-viral transfection, transposons have also been accommodated in conven- tional viral vectors, including adenoviral, ${ }^{47,146,170,171}$ herpes-simplex viral, ${ }^{172} \mathrm{AAV},{ }^{170}$ and integrationdefective lentiviral vectors. ${ }^{173-175}$ Some of these viral vector-based transposon delivery approaches were used for in vivo delivery and resulted in high transduction efficiency and persistent expression in murine ${ }^{146,170,172}$ and canine models. ${ }^{146}$ Nevertheless, these hybrid systems inevitably result in some disadvantages due to the viral vector components, such as potential immunogenicity.

\section{TRANSPOSON-BASED GENE THERAPY IN CLINICAL TRIALS}

There are multiple ongoing clinical trials using $S B$ transposons for ex vivo gene therapy. The first approved transposon-based clinical application was based on early-generation SB technology to generate CAR T cells for adoptive immunotherapy in patients with $\mathrm{CD} 19^{+}$B-lymphoid malignancies (i.e., non-Hodgkin lymphoma and acute lymphoblastic leukemia), spearheaded by Cooper et al. ${ }^{176}$ In an attempt to decrease the relapse rate in these patients, they were injected with autologous transposon-modified CAR T cells along with autologous HSC. Alternatively, patients were treated 
with allogeneic transposon-modified CAR $\mathrm{T}$ cells and allogeneic HSC. ${ }^{173,174}$ The CAR T cells were selectively expanded in vitro $>1,000$-fold, resulting in $80-90 \%$ CAR expressing T cells. This required a protracted expansion of the gene-modified CAR $\mathrm{T}$ cells on an "artificial" antigen-presenting cell line (derived from K562) that expressed CD19, CD86, CD137L, and membrane-bound IL-15. Thirty months after autologous HSC transplantation, progression-free survival (PFS) ${ }^{173}$ was $83 \%$, and overall survival (OS) was $100 \%$. PFS and OS corresponded to $53 \%$ and $63 \%$, respectively, following allogeneic HSC transplantation. This is lower than in the autologous setting, possibly due to the need for immune-suppression to prevent graft-versushost disease (GvHD). Regardless, it would appear than the adoptive transfer of the transposonmodified CAR T cells significantly increased PFS and OS compared to historical controls based on autologous or allogeneic HSC transplantation, without CAR T cell therapy. There was no evidence of cytokine release syndrome, exacerbated GvHD, or other overt toxicities. Nevertheless, mild GvHD was reported in a few allogeneic recipients. The transposon-modified CAR T cells persisted for an average of 201 days in the autologous and 51 days in the allogeneic transplantation setting, with no evidence of integration hotspots of the transposon in the CAR $\mathrm{T}$ cell genome or malignant transformation of the CAR T cells. Control trials are needed to corroborate these encouraging results. Several clinical protocols have been initiated based on the same principle use of CD19-specific CAR T cells that varied based on the origin of the T cells (e.g., autologous, allogeneic, cord blood), cell dose, or lymphodepletion therapy (NCT00968760, NCT01497184, NCT01653717, NCT02529813, and NCT01362452).

One potential caveat of the original CAR $\mathrm{T}$ cell in vitro expansion protocol is that it takes several weeks. Consequently, this protracted cell culture period may potentially compromise the effectiveness of the CAR T cells, at least in part due to cellular exhaustion and/or impaired engraftment of fully functional CAR $\mathrm{T}$ cells. Increasing the overall transposition efficiency by using hyperactive SB100X, mini-circle plasmids or combinations thereof may also require a less prolonged in vitro enrichment period of the transposon-modified CAR T cells. ${ }^{126,177,178}$ Even mini-circle plasmids entirely devoid of antibiotic resistance genes can potentially be used. ${ }^{177}$

Alternatively, co-transfection of the SB transposase construct with two distinct transposons, one encoding CAR and the other mIL15, can potentially shorten the in vitro expansion period. ${ }^{178}$ This is based on the premise that the proliferation of the CAR/mIL15 transposon-modified T cells continues in vivo after transplantation, offering a selective survival advantage. Adoptive transfer of transposon-modified $\mathrm{T}$ cells that express both CAR and mIL15 in CD19 $9^{+}$tumor-bearing mouse models are more effective and persistent longer than the first-generation CAR $\mathrm{T}$ cells. Interestingly, they also display a transcriptional profile and marker gene expression closely resembling that of bona fide memory $\mathrm{T}$ cell,s which would be advantageous with respect to enhancing in vivo persistence of the modified CAR $\mathrm{T}$ cells. It is likely that this CAR T cell concept based on transposon-modified $\mathrm{T}$ cells will benefit from the advances made in lentiviral vector modified CAR $\mathrm{T}$ cells that are now poised to target other lymphohematopoietic malignancies and solid tumors. In addition to using CARmodified T cells, $S B$ transposons have also been explored to express $\mathrm{T}$ cell receptors specific for MHC class I-restricted neoplastic neoantigens that are frequently observed in epithelial malignancies. ${ }^{179}$ Though cancer has so far been the main target disease for transposon-based therapies, this sets the stage for targeting other diseases with transposon-modified cells. In particular, a clinical trial is imminent based on the use of SB100modified pigment epithelial cells expressing antiangiogenic factors to treat age-related macular degeneration (AMD). ${ }^{174,180}$

\section{INSERTIONAL ONCOGENESIS}

Insertional mutagenesis is one of the main concerns using integrative-based gene therapy. Genomic integration may potentially activate oncogenes or interrupt tumor-suppressor genes and hence contribute to malignant transformation. Several studies have been performed to identify the preference of DNA integration sites mediated by transposons. $S B$ transposon appears to integrate at central TA dinucleotide surrounded by palindromic AT repeats, which contribute to bendable DNA conformation. ${ }^{181}$ Target site of $P B$ transposon is known to be TTAA-rich region, regardless of the surrounding AT content, ${ }^{182}$ whereas Tol2 transposon tends to integrate at weak consensus palindromic-like TNA(C/G)TTATAA(G/C)TNA octanucleotides. ${ }^{69,183}$ In terms of genomic integration pattern, $P B$ transposon demonstrated highest integration preference in transcriptional units $(\sim 50 \%$ into RefSeq genes) relative to $S B$ and Tol2 (ranging from $40 \%$ to $45 \%$ into RefSeq genes). Whereas $S B$ seemed integrate quite randomly in the target genome, $P B$ and Tol2 transposons exhibited a stronger 
biased integration toward DNaseI hypersensitivity site, CpG islands, and transcriptional start sites. ${ }^{137}$ Therefore, $S B$ seems to have a more favorable integration profile compared to $P B$ and Tol2 transposons, though this may also be influenced by the cell type. Ultimately, the safety and estimated risk of insertional oncogenesis of a given transposon construct were tested in tumor-prone mouse models. ${ }^{59}$ Minimizing the risk of insertional mutagenesis mediated by transposition is an important priority to move this technology forward. The first strategy is to design the expression cassette itself carefully and avoid the use of elements such as strong viral enhancers, which potentially increase cis-activation of neighboring oncogenes at the integration locus. Alternatively, it is possible to redirect transposon integration toward validated "safeharbor" sites. This can be achieved by transposase modification at $\mathrm{N}$ - or $\mathrm{C}$-terminal regions to carry site-directed DNA binding domain, thereby enabling site-specific transposition at these designated target regions in the genome. ${ }^{184-186}$ From a safety perspective, it is encouraging that the transposon TIRs have only weak enhancer activity, in contrast to the $\gamma$-retroviral vector long terminal repeats that carry a greater risk of insertional oncogenesis. ${ }^{187}$ Nevertheless, to augment the overall safety of transposon further and to minimize the risk of insertional oncogenesis, insulators have been considered. ${ }^{188}$ However, these insulators may also have adverse epigenetic effects or may contribute to vector instability, which would need to be weighed against their potential benefits.

\section{CONCLUSIONS AND PERSPECTIVES}

DNA transposon systems, particularly those based on $S B, P B$, and Tol2, have emerged as promising alternatives to overcome some of the manufacturing constraints associated with the use of viral vectors. Preclinical and clinical studies rely on the transient expression of the transposase enabling stable transposition of the corresponding transposon in the target cell genome. Though initial studies relied on the co-transfection of the transposase-containing expression construct with the transposon containing the gene of interest, subsequent studies showed that transient delivery of the transposase by mRNA would be preferred, since this would minimize the risk of continued transposon remobilization. The original SB and $\mathrm{PB}$ systems have been engineered to augment their overall transposition efficacy further by introducing specific mutations giving rise to the SB100X and hyPB systems that are among the most effi- cient ones for preclinical studies and clinical applications. The DNA transposons are very versatile and have been validated for ex vivo and in vivo gene therapy for many disease targets. Despite their promise, delivery remains a challenge requiring the use of non-viral transfection methods that often result in significant cytotoxicity. Though direct in vivo applications using transposons have often relied on the use of viral vectors, it would be preferred to use a safe and efficient non-viral transfection system instead, given the known immune ramifications associated with the use of viral components. Unfortunately, the overall efficacy of non-viral in vivo transfection is often limited and/or the transfection methods used are not readily amenable for clinical applications (e.g., hydrodynamic transfection). Nevertheless, the development of novel non-viral nanoparticle technologies may open new perspectives for in vivo delivery of transposons. This warrants further investigation in preclinical disease models and requires robust optimized methods for large-scale nanoparticle manufacturing. The use of transposons should also be considered in comparison with gene editing technologies based on designer nucleases (i.e., ZFN, TALEN, CRISPR/Cas9, homing endonucleases). The relative efficacy and safety of transposons versus gene editing should be carefully assessed on a case-by-case basis and depends on several confounding variables, including the disease target, cell type, transfection method, and so on. Nevertheless, for some applications, transposons can be used in combination with these gene editing technologies (e.g., "seamless" gene editing of iPS cells). The use of transposon technology to generate CAR $\mathrm{T}$ cells for cancer immunotherapy constitutes one of the most promising clinical applications to date. Moreover, these advances set the stage for future clinical trials to treat patients suffering from other genetic or acquired diseases.

\section{ACKNOWLEDGMENTS}

We thank the members of the Department of Gene Therapy and Regenerative Medicine and our collaborators for their various contributions to some of the work presented in this review. We also wish to thank FWO, AFM, VUB-IOF-GEAR (GENEFIX), SRP-Groeier, and Willy Gepts for providing financial support. We thank Dr. Chai for critical reading of the manuscript.

\section{AUTHOR DISCLOSURE}

No competing financial interests exist. 


\section{REFERENCES}

1. VandenDriessche T. Gene therapy delivers. Hum Gene Ther 2009;20:1222-1223.

2. Kay MA. State-of-the-art gene-based therapies: the road ahead. Nat Rev Genet 2011;12:316-328.

3. Naldini L. Gene therapy returns to centre stage. Nature 2015;526:351-360.

4. Naldini L. Ex vivo gene transfer and correction for cell-based therapies. Nat Rev Genet 2011;12: 301-315.

5. Ciceri F, Bonini C, Stanghellini MT, et al. Infusion of suicide-gene-engineered donor lymphocytes after family haploidentical haemopoietic stem-cell transplantation for leukaemia (the TK007 trial): a non-randomised Phase I-II study. Lancet Oncol 2009;10:489-500.

6. Aiuti A, Roncarolo MG, Naldini L. Gene therapy for ADA-SCID, the first marketing approval of an ex vivo gene therapy in Europe: paving the road for the next generation of advanced therapy medicinal products. EMBO Mol Med 2017;9: 737-740.

7. Aiuti A, Cattaneo F, Galimberti S, et al. Gene therapy for immunodeficiency due to adenosine deaminase deficiency. N Engl J Med 2009;360: 447-458.

8. Gaudet D, Stroes ES, Methot J, et al. Long-term retrospective analysis of gene therapy with alipogene tiparvovec and its effect on lipoprotein lipase deficiency-induced pancreatitis. Hum Gene Ther 2016;27:916-925.

9. Manno CS, Pierce GF, Arruda VR, et al. Successful transduction of liver in hemophilia by AAV-Factor IX and limitations imposed by the host immune response. Nat Med 2006;12:342347.

10. Hacein-Bey-Abina S, Von Kalle C, Schmidt M, et al. LMO2-associated clonal T cell proliferation in two patients after gene therapy for SCID-X1. Science 2003;302:415-419.

11. Hacein-Bey-Abina S, Garrigue A, Wang GP, et al. Insertional oncogenesis in 4 patients after retrovirus-mediated gene therapy of SCID-X1. J Clin Invest 2008;118:3132-3142.

12. Braun CJ, Boztug K, Paruzynski A, et al. Gene therapy for Wiskott-Aldrich syndrome-longterm efficacy and genotoxicity. Sci Transl Med 2014;6:227ra233.

13. Cavazzana-Calvo M, Payen E, Negre 0 , et al. Transfusion independence and HMGA2 activation after gene therapy of human beta-thalassaemia. Nature 2010;467:318-322.

14. Yin H, Kanasty RL, Eltoukhy AA, et al. Non-viral vectors for gene-based therapy. Nat Rev Genet 2014;15:541-555.

15. VandenDriessche $T$, Ivics $Z$, Izsvak $Z$, et al. Emerging potential of transposons for gene therapy and generation of induced pluripotent stem cells. Blood 2009;114:1461-1468.
16. Ivics $Z$, Izsvak $Z$. The expanding universe of transposon technologies for gene and cell engineering. Mob DNA 2010;1:25.

17. Ivics Z, Izsvak Z. Nonviral gene delivery with the Sleeping Beauty transposon system. Hum Gene Ther 2011;22:1043-1051.

18. Hackett PB Jr, Aronovich EL, Hunter D, et al Efficacy and safety of Sleeping Beauty transposon-mediated gene transfer in preclinical animal studies. Curr Gene Ther 2011;11:341-349.

19. Di Matteo M, Belay E, Chuah MK, et al. Recent developments in transposon-mediated gene therapy. Expert Opin Biol Ther 2012;12:841-858.

20. Yonenaga $Y$, Mori $A$, Fujimoto $A$, et al. The administration of naked plasmid DNA into the liver induces antitumor innate immunity in a murine liver metastasis model. J Gene Med 2007:9:299-307.

21. Yoshinaga $T$, Yasuda $K$, Ogawa $Y$, et al. DNA and its cationic lipid complexes induce $\mathrm{CpG}$ motif-dependent activation of murine dendritic cells. Immunology 2007;120:295-302.

22. Ayarpadikannan S, Kim HS. The impact of transposable elements in genome evolution and genetic instability and their implications in various diseases. Genomics Inform 2014;12:98-104.

23. Pereira V, Enard D, Eyre-Walker A. The effect of transposable element insertions on gene expression evolution in rodents. PLoS One 2009;4: e4321.

24. Goodier JL. Restricting retrotransposons: a review. Mob DNA 2016;7:16

25. Soifer H, Higo C, Kazazian HH Jr, et al. Stable integration of transgenes delivered by a retrotransposon-adenovirus hybrid vector. Hum Gene Ther 2001;12:1417-1428.

26. Cordaux R, Batzer MA. The impact of retrotransposons on human genome evolution. Nat Rev Genet 2009;10:691-703.

27. Pace JK 2nd, Feschotte C. The evolutionary history of human DNA transposons: evidence for intense activity in the primate lineage. Genome Res 2007;17:422-432.

28. Radice AD, Bugaj B, Fitch DH, et al. Widespread occurrence of the Tc1 transposon family: Tc1-like transposons from teleost fish. Mol Gen Genet 1994;244:606-612.

29. Ivics Z, Hackett PB, Plasterk RH, et al. Molecular reconstruction of Sleeping Beauty, a Tc1-like transposon from fish, and its transposition in human cells. Cell 1997:91:501-510.

30. Izsvak Z, Khare D, Behlke J, et al. Involvement of a bifunctional, paired-like DNA-binding domain and a transpositional enhancer in Sleeping Beauty transposition. J Biol Chem 2002;277: 34581-34588.

31. Narayanavari SA, Chilkunda SS, Ivics Z, et al. Sleeping Beauty transposition: from biology to applications. Crit Rev Biochem Mol Biol 2017;52 18-44.

32. Plasterk RH, Izsvak Z, Ivics Z. Resident aliens: the Tc1/mariner superfamily of transposable elements. Trends Genet 1999;15:326-332.

33. Feng JA, Johnson RC, Dickerson RE. Hin recombinase bound to DNA: the origin of specificity in major and minor groove interactions. Science 1994;263:348-355

34. Zayed H, Izsvák Z, Khare D, et al. The DNAbending protein HMGB1 is a cellular cofactor of Sleeping Beauty transposition. Nucleic Acids Res 2003;31:2313-2322.

35. Yant SR, Kay MA. Nonhomologous-end-joining factors regulate DNA repair fidelity during Sleeping Beauty element transposition in mammalian cells. Mol Cell Biol 2003;23:8505-8518.

36. Wilber $A$, Wangensteen $\mathrm{KJ}$, Chen $\mathrm{Y}$, et al Messenger RNA as a source of transposase for Sleeping Beauty transposon-mediated correction of hereditary tyrosinemia type I. Mol Ther 2007 15:1280-1287.

37. Wilber A, Frandsen JL, Geurts JL, et al. RNA as a source of transposase for Sleeping Beauty mediated gene insertion and expression in somatic cells and tissues. Mol Ther 2006;13:625-630

38. Wilber A, Wangensteen KJ, Chen $Y$, et al Messenger RNA as a source of transposase for Sleeping Beauty transposon-mediated correction of hereditary tyrosinemia type I. Mol Ther 2007; 15:1280-1287.

39. Galla M, Schambach A, Falk CS, et al. Avoiding cytotoxicity of transposases by dose-controlled mRNA delivery. Nucleic Acids Res 2011;39:7147-7160.

40. Galla M, Schambach A, Baum C. Retrovirusbased mRNA transfer for transient cell manipulation. Methods Mol Biol 2013;969:139-161.

41. Geurts AM, Yang Y, Clark KJ, et al. Gene transfer into genomes of human cells by the Sleeping Beauty transposon system. Mol Ther 2003;8:108-117.

42. Yant SR, Huang Y, Akache B, et al. Site-directed transposon integration in human cells. Nucleic Acids Res 2007;35:e50.

43. Mates L, Chuah MK, Belay E, et al. Molecular evolution of a novel hyperactive Sleeping Beauty transposase enables robust stable gene transfer in vertebrates. Nat Genet 2009;41:753-761.

44. Loperfido M, Jarmin S, Dastidar S, et al. piggyBac transposons expressing full-length human dystrophin enable genetic correction of dystrophic mesoangioblasts. Nucleic Acids Res 2016; 44:744-760.

45. Belay E, Matrai J, Acosta-Sanchez A, et al. Novel hyperactive transposons for genetic modification of induced pluripotent and adult stem cells: a nonviral paradigm for coaxed differentiation. Stem Cells 2010;28:1760-1771. 
46. Zhang W, Solanki M, Muther N, et al. Hybrid adeno-associated viral vectors utilizing transposasemediated somatic integration for stable transgene expression in human cells. PLoS One 2013;8: e76771.

47. Boehme P, Zhang W, Solanki M, et al. A highcapacity adenoviral hybrid vector system utilizing the hyperactive Sleeping Beauty transposase SB100X for enhanced integration. Mol Ther Nucleic Acids 2016;5:e337.

48. Zayed H, Izsvak Z, Walisko O, et al. Development of hyperactive Sleeping Beauty transposon vectors by mutational analysis. Mol Ther 2004;9: 292-304.

49. de Silva S, Mastrangelo MA, Lotta LT Jr, et al. Extending the transposable payload limit of Sleeping Beauty (SB) using the herpes simplex virus (HSV)/SB amplicon-vector platform. Gene Ther 2010;17:424-431.

50. Potter KN, Faulkner P, MacKinnon EA. Strain selection during serial passage of Trichoplusia in nuclear polyhedrosis virus. J Virol 1976;18:10401050.

51. Fraser MJ, Hink WF. The isolation and characterization of the MP and FP plaque variants of Galleria mellonella nuclear polyhedrosis virus. Virology 1982;117:366-378.

52. Yusa K. piggyBac transposon. Microbiol Spectr 2015;3:MDNA3-0028-2014.

53. Yuan Y-W, Wessler SR. The catalytic domain of all eukaryotic cut-and-paste transposase superfamilies. Proc Natl Acad Sci U S A 2011;108: 7884-7889.

54. Hickman $A B$, Chandler M, Dyda F. Integrating prokaryotes and eukaryotes: DNA transposases in light of structure. Crit Rev Biochem Mol Biol 2010;45:50-69

55. Mitra R, Fain-Thornton J, Craig NL. piggyBac can bypass DNA synthesis during cut and paste transposition. EMBO J 2008;27:1097-1109.

56. Lacoste A, Berenshteyn F, Brivanlou AH. An efficient and reversible transposable system for gene delivery and lineage-specific differentiation in human embryonic stem cells. Cell Stem Cell 2009;5:332-342.

57. Cadinanos J, Bradley A. Generation of an inducible and optimized piggyBac transposon system. Nucleic Acids Res 2007;35:e87.

58. Yusa K, Zhou L, Li MA, et al. A hyperactive piggyBac transposase for mammalian applications. Proc Natl Acad Sci U S A 2011;108:1531-1536.

59. Di Matteo M, Samara-Kuko E, Ward NJ, et al. Hyperactive piggyBac transposons for sustained and robust liver-targeted gene therapy. Mol Ther 2014;22:1614-1624.

60. Zhang G, Budker V, Williams P, et al. Efficient expression of naked DNA delivered intraarterially to limb muscles of nonhuman primates. Hum Gene Ther 2001;12:427-438.

61. Meir YJ, Weirauch MT, Yang HS, et al. Genomewide target profiling of piggyBac and Tol2 in
HEK 293: pros and cons for gene discovery and gene therapy. BMC Biotechnol 2011;11:28.

62. Di Matteo M, Samara-Kuko E, Ward NJ, et al. Hyperactive piggyBac transposons for sustained and robust liver-targeted gene therapy. Mol Ther 2014;22:1614-1624.

63. Li MA, Turner DJ, Ning Z, et al. Mobilization of giant piggyBac transposons in the mouse genome. Nucleic Acids Res 2011;39:e148.

64. Katter K, Geurts AM, Hoffmann 0, et al. Transposon-mediated transgenesis, transgenic rescue, and tissue-specific gene expression in rodents and rabbits. FASEB J 2013;27:930-941.

65. Di Matteo M, Matrai J, Belay E, et al. PiggyBac toolbox. Methods Mol Biol 2012;859:241-254.

66. Belay E, Dastidar S, VandenDriessche T, et al. Transposon-mediated gene transfer into adult and induced pluripotent stem cells. Curr Gene Ther 2011;11:406-413.

67. Feschotte C. The piggyBac transposon holds promise for human gene therapy. Proc Natl Acad Sci U S A 2006;103:14981-14982.

68. Koga A, Suzuki M, Inagaki H, et al. Transposable element in fish. Nature 1996;383:30.

69. Ni J, Wangensteen KJ, Nelsen D, et al. Active recombinant Tol2 transposase for gene transfer and gene discovery applications. Mob DNA 2016;7:6.

70. Kawakami K, Shima A, Kawakami N. Identification of a functional transposase of the Tol2 element, an Ac-like element from the Japanese medaka fish, and its transposition in the zebrafish germ lineage. Proc Natl Acad Sci U S A 2000;97:11403-11408.

71. Urasaki A, Morvan G, Kawakami K. Functional dissection of the Tol2 transposable element identified the minimal cis-sequence and a highly repetitive sequence in the subterminal region essential for transposition. Genetics 2006;174: 639-649.

72. Balciunas D, Wangensteen KJ, Wilber A, et al. Harnessing a high cargo-capacity transposon for genetic applications in vertebrates. PLoS Genet 2006;2:e169.

73. Tsukahara T, Iwase N, Kawakami K, et al. The Tol2 transposon system mediates the genetic engineering of T-cells with CD19-specific chimeric antigen receptors for B-cell malignancies. Gene Ther 2015;22:209-215.

74. Muliawan HS, Nakayama K, Yagi K, et al. Stable somatic gene expression in mouse lungs following electroporation-mediated Tol2 transposon delivery. Kobe J Med Sci 2015;61:E47-53.

75. Yang $Y$, Wang $W$, Huang $T$, et al. Transgenesis of Tol2-mediated seamlessly constructed BAC mammary gland expression vectors in Mus musculus. J Biotechnol 2016;218:66-72.

76. Sumiyama K, Kawakami K, Yagita K. A simple and highly efficient transgenesis method in mice with the Tol2 transposon system and cytoplasmic microinjection. Genomics 2010;95:306-311.
77. Suster ML, Sumiyama K, Kawakami K. Transposon-mediated BAC transgenesis in zebrafish and mice. BMC Genomics 2009;10:477.

78. Hamlet MR, Yergeau DA, Kuliyev E, et al. Tol2 transposon-mediated transgenesis in Xenopus tropicalis. Genesis 2006;44:438-445.

79. Sato Y, Kasai T, Nakagawa S, et al. Stable integration and conditional expression of electroporated transgenes in chicken embryos. Dev Biol 2007;305:616-624.

80. Urasaki A, Asakawa K, Kawakami K. Efficient transposition of the Tol2 transposable element from a single-copy donor in zebrafish. Proc Natl Acad Sci U S A 2008;105:19827-19832.

81. Suster ML, Abe G, Schouw A, et al. Transposonmediated BAC transgenesis in zebrafish. Nat Protoc 2011;6:1998-2021.

82. Keng VW, Ryan BJ, Wangensteen KJ, et al. Efficient transposition of Tol2 in the mouse germline. Genetics 2009;183:1565-1573.

83. Grabundzija I, Irgang M, Mates L, et al. Comparative analysis of transposable element vector systems in human cells. Mol Ther 2010;18:12001209.

84. Turchiano G, Latella MC, Gogol-Doring A, et al. Genomic analysis of Sleeping Beauty transposon integration in human somatic cells. PLoS One 2014;9:e112712.

85. Converse AD, Belur LR, Gori JL, et al. Counterselection and co-delivery of transposon and transposase functions for Sleeping Beautymediated transposition in cultured mammalian cells. Biosci Rep 2004;24:577-594.

86. Li Z, Michael IP, Zhou D, et al. Simple piggyBac transposon-based mammalian cell expression system for inducible protein production. Proc Natl Acad Sci U S A 2013;110:5004-5009.

87. Glover JD, Taylor L, Sherman A, et al. A novel piggyBac transposon inducible expression system identifies a role for AKT signalling in primordial germ cell migration. PLoS One 2013;8: e77222.

88. Bire S, Gosset D, Jegot G, et al. Exogenous mRNA delivery and bioavailability in gene transfer mediated by piggyBac transposition. BMC Biotechnol 2013;13:75.

89. Ley D, Van Zwieten R, Puttini S, et al. A piggyBac-mediated approach for muscle gene transfer or cell therapy. Stem Cell Res 2014;13: 390-403.

90. Hollis RP, Nightingale SJ, Wang $X$, et al. Stable gene transfer to human CD34(+) hematopoietic cells using the Sleeping Beauty transposon. Exp Hematol 2006;34:1333-1343.

91. Sumiyoshi T, Holt NG, Hollis RP, et al. Stable transgene expression in primitive human CD34+ hematopoietic stem/progenitor cells, using the Sleeping Beauty transposon system. Hum Gene Ther 2009;20:1607-1626.

92. Xue $X$, Huang $X$, Nodland $S E$, et al. Stable gene transfer and expression in cord blood-derived 
CD34+ hematopoietic stem and progenitor cells by a hyperactive Sleeping Beauty transposon system. Blood 2009;114:1319-1330.

93. Sjeklocha LM, Wong PY, Belcher JD, et al. betaGlobin Sleeping Beauty transposon reduces red blood cell sickling in a patient-derived CD34(+)based in vitro model. PLoS One 2013;8:e80403.

94. Baroffio A, Hamann M, Bernheim L, et al. Identification of self-renewing myoblasts in the progeny of single human muscle satellite cells. Differentiation 1996;60:47-57.

95. Joyce NC, Oskarsson B, Jin LW. Muscle biopsy evaluation in neuromuscular disorders. Phys Med Rehabil Clin N Am 2012;23:609-631.

96. Escobar H, Schowel V, Spuler S, et al. Full-length dysferlin transfer by the hyperactive Sleeping Beauty transposase restores dysferlin-deficient muscle. Mol Ther Nucleic Acids 2016;5:e277.

97. Muses S, Morgan JE, Wells DJ. Restoration of dystrophin expression using the Sleeping Beauty transposon. Version 2. PLoS Curr 2011;3:RRN1296.

98. Troyanovsky B, Bitko V, Pastukh V, et al. The functionality of minimal piggyBac transposons in mammalian cells. Mol Ther Nucleic Acids 2016:5:e369

99. Ma K, Wang D-D, Lin Y, et al. Synergetic targeted delivery of Sleeping-Beauty transposon system to mesenchymal stem cells using LPD nanoparticles modified with a phage-displayed targeting peptide. Adv Funct Mater 2013;23 1172-1181.

100. Myers TJ, Granero-Molto F, Longobardi L, et al. Mesenchymal stem cells at the intersection of cell and gene therapy. Expert Opin Biol Ther 2010;10:1663-1679.

101. Wen S, Zhang H, Li Y, et al. Characterization of constitutive promoters for piggyBac transposonmediated stable transgene expression in mesenchymal stem cells (MSCs). PLoS One 2014;9: e94397.

102. Chen X, Cui J, Yan Z, et al. Sustained high level transgene expression in mammalian cells mediated by the optimized piggyBac transposon system. Genes Dis 2015;2:96-105.

103. Bahrambeigi V, Ahmadi N, Moisyadi S, et al. PhiC31/PiggyBac modified stromal stem cells: effect of interferon $\gamma$ and/or tumor necrosis factor (TNF)-related apoptosis-inducing ligand (TRAIL) on murine melanoma. Mol Cancer 2014;13.

104. Minasi MG, Riminucci M, Angelis LD, et al. The meso-angioblast: a multipotent, self-renewing cell that originates from the dorsal aorta and differentiates into most mesodermal tissues. Development 2002;129:2773-2783.

105. Sampaolesi M, Blot S, D'Antona G, et al. Mesoangioblast stem cells ameliorate muscle function in dystrophic dogs. Nature 2006;444: 574-579.

106. Domi T, Porrello E, Velardo D, et al. Mesoangioblast delivery of miniagrin ameliorates murine model of merosin-deficient congenital muscular dystrophy type 1A. Skelet Muscle 2015;5:30.

107. Takahashi K, Yamanaka S. Induction of pluripotent stem cells from mouse embryonic and adult fibroblast cultures by defined factors. Cell 2006 126:663-676.

108. Grabundzija I, Wang J, Sebe A, et al. Sleeping Beauty transposon-based system for cellular reprogramming and targeted gene insertion in induced pluripotent stem cells. Nucleic Acids Res 2013;41:1829-1847.

109. Talluri TR, Kumar D, Glage S, et al. Non-viral reprogramming of fibroblasts into induced pluripotent stem cells by Sleeping Beauty and piggyBac transposons. Biochem Biophys Res Commun 2014:450:581-587.

110. Woltjen K, Michael IP, Mohseni P, et al. piggy$B a c$ transposition reprograms fibroblasts to induced pluripotent stem cells. Nature 2009;458: 766-770.

111. Talluri TR, Kumar D, Glage $S$, et al. Derivation and characterization of bovine induced pluripotent stem cells by transposon-mediated reprogramming. Cell Reprogram 2015;17:131-140.

112. Kawaguchi T, Tsukiyama T, Kimura K, et al. Generation of naïve bovine induced pluripotent stem cells using piggyBac transposition of doxycyclineinducible transcription factors. PLoS One 2015;10.

113. Kues WA, Herrmann D, Barg-Kues B, et al. Derivation and characterization of Sleeping Beauty transposon-mediated porcine induced pluripotent stem cells. Stem Cells Dev 2013;22:124-135.

114. Davis RP, Nemes C, Varga E, et al. Generation of induced pluripotent stem cells from human foeta fibroblasts using the Sleeping Beauty transposon gene delivery system. Differentiation 2013;86: 30-37.

115. Woltjen $K$, Hämäläinen $R$, Kibschull $M$, et al. Transgene-free production of pluripotent stem cells using piggyBac transposons. Methods $\mathrm{Mol}$ Biol 2011;767:87-103.

116. Wang G, Yang L, Grishin D, et al. Efficient, footprint-free human iPSC genome editing by consolidation of Cas9/CRISPR and piggyBac technologies. Nat Protoc 2017:12:88-103.

117. Filareto A, Parker S, Darabi R, et al. An ex vivo gene therapy approach to treat muscular dystrophy using inducible pluripotent stem cells. Nat Commun 2013;4:1549.

118. Tanaka A, Woltjen K, Miyake K, et al. Efficient and reproducible myogenic differentiation from human iPS cells: prospects for modeling Miyoshi myopathy in vitro. PLoS One 2013;8:e61540.

119. Yusa K. Seamless genome editing in human pluripotent stem cells using custom endonucleasebased gene targeting and the piggyBac transposon. Nat Protoc 2013;8:2061-2078.

120. Sun N, Zhao H. Seamless correction of the sickle cell disease mutation of the HBB gene in human induced pluripotent stem cells using TALENs Biotechnol Bioeng 2014;111:1048-1053.
121. Xie F, Ye L, Chang JC, et al. Seamless gene correction of beta-thalassemia mutations in patient-specific iPSCs using CRISPR/Cas9 and piggyBac. Genome Res 2014;24:1526-1533.

122. Xu X, Tay Y, Sim B, et al. Reversal of phenotypic abnormalities by CRISPR/Cas9-mediated gene correction in Huntington disease patient-derived induced pluripotent stem cells. Stem Cell Reports 2017:8:619-633.

123. Lim WA, June $\mathrm{CH}$. The principles of engineering immune cells to treat cancer. Cell 2017:168:724740.

124. Huang X, Guo H, Kang J, et al. Sleeping Beauty transposon-mediated engineering of human primary $T$ cells for therapy of CD19+ lymphoid malignancies. Mol Ther 2008;16:580-589.

125. Singh H, Manuri PR, Olivares $S$, et al. Redirecting specificity of T-cell populations for CD19 using the Sleeping Beauty system. Cancer Res 2008; 68:2961-2971.

126. Jin Z, Maiti S, Huls $H$, et al. The hyperactive Sleeping Beauty transposase SB100X improves the genetic modification of $T$ cells to express a chimeric antigen receptor. Gene Ther 2011;18: 849-856.

127. Thokala R, Olivares $S, M i T$, et al. Redirecting specificity of T cells using the Sleeping Beauty system to express chimeric antigen receptors by mix-and-matching of $\mathrm{VL}$ and $\mathrm{VH}$ domains targeting CD123+ tumors. PLoS One 2016;11.

128. Huang X, Park H, Greene J, et al. IGF1R- and ROR1-specific CAR T cells as a potential therapy for high risk sarcomas. PLoS One 2015;10.

129. Deniger DC, Yu J, Huls MH, et al. Sleeping Beauty transposition of chimeric antigen receptors targeting receptor tyrosine kinase-like orphan receptor-1 (ROR1) into diverse memory T-cell populations. PLoS One 2015;10.

130. Huang G, Yu L, Cooper LJ, et al. Genetically modified $T$ cells targeting interleukin-11 receptor alpha-chain kill human osteosarcoma cells and induce the regression of established osteosarcoma lung metastases. Cancer Res 2012;72:271-281.

131. Dolnikov A, Shen S, Klamer G, et al. Antileukemic potency of CD19-specific T cells against chemoresistant pediatric acute lymphoblastic leukemia. Exp Hematol 2015:43:1001-1014.e1005.

132. Saito S, Nakazawa Y, Sueki A, et al. Antileukemic potency of piggyBac-mediated CD19specific $T$ cells against refractory Philadelphia chromosome-positive acute lymphoblastic leukemia. Cytotherapy 2014;16:1257-1269

133. Manuri PV, Wilson MH, Maiti SN, et al. piggyBac transposon/transposase system to generate CD19-specific $T$ cells for the treatment of Blineage malignancies. Hum Gene Ther 2010;21: 427-437.

134. Nakazawa Y, Matsuda K, Kurata T, et al. Antiproliferative effects of $T$ cells expressing a ligand-based chimeric antigen receptor against CD116 on CD34(+) cells of juvenile myelomonocytic leukemia. J Hematol Oncol 2016;9:27. 
135. Nakazawa $Y$, Huye LE, Salsman VS, et al. PiggyBac-mediated cancer immunotherapy using EBV-specific cytotoxic T-cells expressing HER2specific chimeric antigen receptor. Mol Ther 2011;19:2133-2143.

136. Xu JY, Ye ZL, Jiang DQ, et al. Mesothelintargeting chimeric antigen receptor-modified T cells by piggyBac transposon system suppress the growth of bile duct carcinoma. Tumour Biol 2017;39:1010428317695949.

137. Huang X, Guo H, Tammana S, et al. Gene transfer efficiency and genome-wide integration profiling of Sleeping Beauty, Tol2, and piggyBac transposons in human primary T cells. Mol Ther 2010;18:1803-1813.

138. Bonamassa B, Hai L, Liu D. Hydrodynamic gene delivery and its applications in pharmaceutical research. Pharm Res 2011;28:694-701.

139. Woodard LE, Cheng J, Welch RC, et al. Kidneyspecific transposon-mediated gene transfer in vivo. Sci Rep 2017;7:44904.

140. Kren BT, Ghosh SS, Linehan CL, et al. Hepatocyte-targeted delivery of Sleeping Beauty mediates efficient gene transfer in vivo. Gene Ther Mol Biol Vol 2003;7:229-238.

141. Yant SR, Meuse L, Chiu W, et al. Somatic integration and long-term transgene expression in normal and haemophilic mice using a DNA transposon system. Nat Genet 2000;25:35-41.

142. Yant SR, Meuse L, Chiu W, et al. Somatic integration and long-term transgene expression in normal and haemophilic mice using a DNA transposon system. Nat Genet 2000;25:35-41.

143. Saridey SK, Liu L, Doherty JE, et al. PiggyBac transposon-based inducible gene expression in vivo after somatic cell gene transfer. Mol Ther 2009;17:2115-2120.

144. Liu L, Liu H, Mah C, et al. Indoleamine 2,3dioxygenase attenuates inhibitor development in gene-therapy-treated hemophilia A mice. Gene Ther 2009;16:724-733.

145. Liu L, Mah C, Fletcher BS. Sustained FVIII expression and phenotypic correction of hemophilia A in neonatal mice using an endothelial-targeted Sleeping Beauty transposon. Mol Ther 2006;13: 1006-1015.

146. Hausl MA, Zhang W, Muther N, et al. Hyperactive Sleeping Beauty transposase enables persistent phenotypic correction in mice and a canine model for hemophilia B. Mol Ther 2010; 18:1896-1906.

147. Belcher JD, Vineyard JV, Bruzzone CM, et al. Heme oxygenase-1 gene delivery by Sleeping Beauty inhibits vascular stasis in a murine model of sickle cell disease. J Mol Med (Berl) 2010;88: 665-675.

148. Aronovich EL, Bell JB, Belur LR, et al. Prolonged expression of a lysosomal enzyme in mouse liver after Sleeping Beauty transposon-mediated gene delivery: implications for non-viral gene therapy of mucopolysaccharidoses. J Gene Med 2007;9: 403-415.
149. Aronovich EL, Bell JB, Khan SA, et al. Systemic correction of storage disease in MPS I NOD/ SCID mice using the Sleeping Beauty transposon system. Mol Ther 2009 17:1136-1144.

150. Hackett P. Non-viral gene therapy by liverdirected hydrodynamic delivery of Sleeping Beauty transposons to treat MPS in dogs. Mol Genet Metab 2015;114:S49-S50.

151. Montini E, Held PK, Noll M, et al. In vivo correction of murine tyrosinemia type I by DNA-mediated transposition. Mol Ther 2002;6:759-769.

152. He CX, Shi D, Wu WJ, et al. Insulin expression in livers of diabetic mice mediated by hydrodynamicsbased administration. World J Gastroenterol 2004; 10:567-572.

153. Turunen TA, Kurkipuro J, Heikura $T$, et al. Sleeping Beauty transposon vectors in liverdirected gene delivery of LDLR and VLDLR for gene therapy of familial hypercholesterolemia. Mol Ther 2016;24:620-635.

154. Wang X, Sarkar DP, Mani P, et al. Long-term reduction of jaundice in Gunn rats by nonviral liver-targeted delivery of Sleeping Beauty transposon. Hepatology 2009;50:815-824.

155. Ohlfest JR, Frandsen JL, Fritz S, et al. Phenotypic correction and long-term expression of factor VIII in hemophilic mice by immunotolerization and nonviral gene transfer using the Sleeping Beauty transposon system. Blood 2005;105:2691-2698.

156. Aronovich EL, Hall BC, Bell JB, et al. Quantitative analysis of alpha-L-iduronidase expression in immunocompetent mice treated with the Sleeping Beauty transposon system. PLoS One 2013;8:e78161.

157. Doherty JE, Huye LE, Yusa K, et al. Hyperactive piggyBac gene transfer in human cells and in vivo. Hum Gene Ther 2012;23:311-320.

158. Johnson BA, Chauhan AK, Staber JM. Long-term expression of Von Willebrand Factor via piggybacmediated gene transfer. Blood 2016;128:3511.

159. Liang M, Woodard LE, Liang $A$, et al. Protective role of insulin-like growth factor-1 receptor in endothelial cells against unilateral ureteral obstruction-induced renal fibrosis. Am J Pathol 2015;185:1234-1250.

160. Liang A, Wang Y, Woodard LE, et al. Loss of glutathione $\mathrm{S}$-transferase A4 accelerates obstruction-induced tubule damage and renal fibrosis. J Pathol 2012;228:448-458.

161. Noda M, Matsui H, Matsunari $Y$, et al. Novel gene therapy strategy for hemophilia A by hydrodynamic gene delivery combined with nonviral piggyBac transposon vector in canine model. Blood 2015;126:4419.

162. Matsui H, Fujimoto N, Sasakawa N, et al. Delivery of full-length factor VIII using a piggyBac transposon vector to correct a mouse model of hemophilia A. PLoS One 2014;9.

163. Staber JM, Pollpeter MJ, Arensdorf A, et al. piggyBac-mediated phenotypic correction of factor VIII deficiency. Mol Ther Methods Clin Dev 2014;1:14042.
164. Alino SF, Herrero MJ, Noguera I, et al. Pig liver gene therapy by noninvasive interventionist catheterism. Gene Ther 2007;14:334-343.

165. Hyland KA, Aronovich EL, Olson ER, et al. Transgene expression in dogs after liver-directed hydrodynamic delivery of Sleeping Beauty transposons using balloon catheters. Hum Gene Ther 2017;28:541-550.

166. Aronovich EL, Hyland KA, Hall BC, et al. Prolonged expression of secreted enzymes in dogs after liver-directed delivery of Sleeping Beauty transposons: implications for non-viral gene therapy of systemic disease. Hum Gene Ther 2017;28:551-564.

167. Podetz-Pedersen KM, Bell JB, Steele TW, et al. Gene expression in lung and liver after intravenous infusion of polyethylenimine complexes of Sleeping Beauty transposons. Hum Gene Ther 2010;21:210-220.

168. Smith TT, Stephan SB, Moffett HF, et al. In situ programming of leukaemia-specific $T$ cells using synthetic DNA nanocarriers. Nat Nanotechnol 2017;12:813-820.

169. Kren BT, Unger GM, Sjeklocha L, et al. Nanocapsule-delivered Sleeping Beauty mediates therapeutic Factor VIII expression in liver sinusoidal endothelial cells of hemophilia $A$ mice. J Clin Invest 2009;119:2086-2099.

170. Cooney AL, Singh BK, Sinn PL. Hybrid nonviral/ viral vector systems for improved piggyBac DNA transposon in vivo delivery. Mol Ther 2015;23:667-674.

171. Yant SR, Ehrhardt A, Mikkelsen JG, et al. Transposition from a gutless adeno-transposon vector stabilizes transgene expression in vivo. Nat Biotechnol 2002;20:999-1005.

172. de Silva S, Mastrangelo MA, Louis T. Lotta J, et al. Herpes simplex virus/Sleeping Beauty vector-based embryonic gene transfer using the HSB5 mutant: loss of apparent transposition hyperactivity in vivo. Hum Gene Ther 2010;21: 1603-1613.

173. Vink CA, Gaspar HB, Gabriel R, et al. Sleeping Beauty transposition from nonintegrating lentivirus. Mol Ther 2009;17:1197-1204.

174. Staunstrup NH, Moldt B, Mates L, et al. Hybrid lentivirus-transposon vectors with a random integration profile in human cells. Mol Ther 2009;17:1205-1214.

175. Moldt B, Miskey C, Staunstrup NH, et al. Comparative genomic integration profiling of Sleeping Beauty transposons mobilized with high efficacy from integrase-defective lentiviral vectors in primary human cells. Mol Ther 2011;19: 1499-1510.

176. Kebriaei P, Singh $H_{\text {, Huls } M H}$, et al. Phase I trials using Sleeping Beauty to generate CD19-specific CAR T cells. J Clin Invest 2016;126:3363-3376.

177. Monjezi R, Miskey C, Gogishvili T, et al. Enhanced CAR T-cell engineering using non-viral Sleeping Beauty transposition from minicircle vectors. Leukemia 2017;31:186-194. 
178. Sharma N, Cai Y, Bak R0, et al. Efficient Sleeping Beauty DNA transposition from DNA minicircles. Mol Ther Nucleic Acids 2013;2:e74.

179. Deniger DC, Pasetto A, Tran E, et al. Stable, nonviral expression of mutated tumor neoantigenspecific T-cell receptors using the Sleeping Beauty transposon/transposase system. Mol Ther 2016; 24:1078-1089.

180. Thumann G, Harmening N, Prat-Souteyrand C, et al. Engineering of PEDF-expressing primary pigment epithelial cells by the SB transposon system delivered by pFAR4 plasmids. Mol Ther Nucleic Acids 2017;6:302-314.

181. Vigdal TJ, Kaufman CD, Izsvak Z, et al. Common physical properties of DNA affecting target site selection of Sleeping Beauty and other Tc1/ mariner transposable elements. J Mol Biol 2002; 323:441-452.

182. Galvan DL, Nakazawa Y, Kaja A, et al. Genomewide mapping of piggyBac transposon integrations in primary human $\mathrm{T}$ cells. $\mathrm{J}$ Immunother 2009;32:837-844.

183. Kondrychyn I, Garcia-Lecea M, Emelyanov A, et al. Genome-wide analysis of Tol2 transposon reintegration in zebrafish. BMC Genomics 2009; 10:418.

184. Wu SC-Y, Meir Y-JJ, Coates CJ, et al. piggyBac is a flexible and highly active transposon as compared to Sleeping Beauty, Tol2, and Mos1 in mammalian cells. 2006;103:15008-15013.

185. Kettlun C, Galvan DL, George AL Jr, et al. Manipulating piggyBac transposon chromosomal integration site selection in human cells. Mol Ther 2011;19:1636-1644.

186. Owens JB, Urschitz J, Stoytchev I, et al. Chimeric piggyBac transposases for genomic targeting in human cells. Nucleic Acids Res 2012;40:69786991.

187. Moldt B, Yant SR, Andersen PR, et al. Cis-acting gene regulatory activities in the terminal regions of Sleeping Beauty DNA transposon-based vectors. Hum Gene Ther 2007;18:1193-1204.

188. Walisko O, Schorn A, Rolfs F, et al. Transcriptional activities of the Sleeping Beauty transposon and shielding its genetic cargo with insulators. Mol Ther 2008;16:359-369.

189. Sjeklocha LM, Park CW, Wong PY, et al. Erythroid-specific expression of beta-globin from Sleeping Beauty-transduced human hematopoietic progenitor cells. PLoS One 2011;6:e29110.

190. Hyland KA, Olson ER, Clark KJ, et al. Sleeping Beauty-mediated correction of Fanconi anemia type C. J Gene Med 2011;13:462-469.

191. Martin PKM, Stilhano RS, Samoto VY, et al. Mesenchymal stem cells do not prevent antibody responses against human $\alpha$-L-iduronidase when used to treat mucopolysaccharidosis type I. PLoS One 2014;9:e92420.
192. Eyjolfsdottir H, Eriksdotter M, Linderoth B, et al. Targeted delivery of nerve growth factor to the cholinergic basal forebrain of Alzheimer's disease patients: application of a second-generation encapsulated cell biodelivery device. Alzheimers Res Ther 2016;8:30.

193. Fjord-Larsen L, Kusk P, Emerich DF, et al. Increased encapsulated cell biodelivery of nerve growth factor in the brain by transposonmediated gene transfer. Gene Ther 2012;19: 1010-1017.

194. Chen ZJ, Kren BT, Wong PY, et al. Sleeping Beauty-mediated down-regulation of huntingtin expression by RNA interference. Biochem Biophys Res Commun 2005;329:646-652.

195. Ortiz-Urda S, Lin Q, Yant SR, et al. Sustainable correction of junctional epidermolysis bullosa via transposon-mediated nonviral gene transfer. Gene Ther 2003;10:1099-1104.

196. Latella MC, Cocchiarella F, De Rosa L, et al. Correction of recessive dystrophic epidermolysis bullosa by transposon-mediated integration of COL7A1 in transplantable patient-derived primary keratinocytes. J Invest Dermatol 2017;137: 836-844.

197. Filareto A, Rinaldi F, Arpke RW, et al. Pax3induced expansion enables the genetic correction of dystrophic satellite cells. Skelet Muscle 2015;5:36

198. Song J, Kim C, Ochoa ER. Sleeping Beautymediated suicide gene therapy of hepatocellular carcinoma. Biosci Biotechnol Biochem 2009;73: 165-168.

199. Vercellotti GM, Khan FB, Nguyen J, et al. Hferritin ferroxidase induces cytoprotective pathways and inhibits microvascular stasis in transgenic sickle mice. Front Pharmacol 2014; 5:79.

200. Vercellotti GM, Zhang P, Nguyen J, et al. Hepatic overexpression of hemopexin inhibits inflammation and vascular stasis in murine models of sickle cell disease. Mol Med 2016;22.

201. Mikkelsen JG, Yant SR, Meuse L, et al. Helperindependent Sleeping Beauty transposontransposase vectors for efficient nonviral gene delivery and persistent gene expression in vivo. Mol Ther 2003;8:654-665.

202. Zhang W, Muck-Hausl M, Wang J, et al. Integration profile and safety of an adenovirus hybrid-vector utilizing hyperactive Sleeping Beauty transposase for somatic integration. PLoS One 2013;8:e75344.

203. Pan X-J, Ma Z-Z, Zhang Q-J, et al. Sleeping Beauty transposon system is a reliable gene delivery tool for hereditary tyrosinaemia type 1 disease gene therapy: size of the foreign gene decides the timing of stable integration into the host chromosomes. J Int Med Res 2012;40: 1850-1859.
204. Liu H, Liu L, Fletcher BS, et al. Sleeping Beautybased gene therapy with indoleamine 2,3dioxygenase inhibits lung allograft fibrosis. FASEB J 2006;20:2384-2386.

205. Xiao J, Meng XM, Huang XR, et al. miR-29 inhibits bleomycin-induced pulmonary fibrosis in mice. Mol Ther 2012;20:1251-1260.

206. Xiao Y, Christou H, Liu L, et al. Endothelial indoleamine 2,3-dioxygenase protects against development of pulmonary hypertension. Am J Respir Crit Care Med 2013;188:482-491.

207. Liu L, Liu H, Visner G, et al. Sleeping Beautymediated eNOS gene therapy attenuates monocrotaline-induced pulmonary hypertension in rats. FASEB J 2006;20:2594-2596.

208. Verhenne S, Vandeputte N, Pareyn I, et al. Longterm prevention of congenital thrombotic thrombocytopenic purpura in ADAMTS13 knockout mice by Sleeping Beauty transposon-mediated gene therapy. Arterioscler Thromb Vasc Biol 2017;37: 836-844.

209. Belur LR, Podetz-Pedersen KM, Sorenson BS, et al. Inhibition of angiogenesis and suppression of colorectal cancer metastatic to the liver using the Sleeping Beauty transposon system. Mol Cancer 2011;10:14.

210. Wu A, Oh S, Ericson K, et al. Transposon-based interferon gamma gene transfer overcomes limitations of episomal plasmid for immunogene therapy of glioblastoma. Cancer Gene Ther 2007; 14:550-560

211. Yusa K, Rashid ST, Strick-Marchand H, et al. Targeted gene correction of alpha1-antitrypsin deficiency in induced pluripotent stem cells. Nature 2011;478:391-394.

212. Ramanayake S, Bilmon I, Bishop D, et al. Lowcost generation of Good Manufacturing Practicegrade CD19-specific chimeric antigen receptorexpressing T cells using piggyBac gene transfer and patient-derived materials. Cytotherapy 2015; 17:1251-1267.

213. Anderson CD, Moisyadi S, Avelar A, et al. Ultrasound-targeted hepatic delivery of factor IX in hemophiliac mice. Gene Ther 2016;23:510-519.

214. Kang $Y$, Zhang $X$, Jiang $W$, et al. Tumor-directed gene therapy in mice using a composite nonviral gene delivery system consisting of the piggyBac transposon and polyethylenimine. BMC Cancer 2009;9:126

215. Kang Y, Yu W, Sun Q, et al. High-level transgene expression mediated by the piggyBac transposon enhances transgenic therapeutic effects in cervical cancer xenografts. Oncol Rep 2010;24:897-907.

Received for publication July 26, 2017; accepted after revision August 22, 2017.

Published online: August 22, 2017. 